\title{
THE SHARP QUANTITATIVE ISOCAPACITARY INEQUALITY (THE CASE OF $p$-CAPACITY)
}

\author{
EKATERINA MUKOSEEVA
}

\begin{abstract}
We prove a sharp quantitative form of isocapacitary inequality in the case of a general $p$. This work is a generalization of the author's paper with Guido De Philippis and Michele Marini, where we treated the case of 2-capacity.
\end{abstract}

\section{INTRODUCTION}

1.1. Background. Let $\Omega \subset \mathbb{R}^{N}$ be an open set. We define the p-capacity of $\Omega$ as

$$
\operatorname{cap}_{p}(\Omega)=\inf _{u \in C_{c}^{\infty}\left(\mathbb{R}^{N}\right)}\left\{\int_{\mathbb{R}^{N}}|\nabla u|^{p} d x: u \geq 1 \text { on } \Omega\right\}
$$

for $1<p<n$. It is easy to see that for problem (1.1) there exists a unique function ${ }^{1}$ $u \in D^{1, p}\left(\mathbb{R}^{n}\right)$ called capacitary potential of $\Omega$ such that

$$
\int_{\mathbb{R}^{N}}|\nabla u|^{p}=\operatorname{cap}_{p}(\Omega)
$$

Moreover, it satisfies the Euler-Lagrange equation (if $\Omega$ is sufficiently smooth, e.g. Lipschitz):

$$
\left\{\begin{array}{l}
\operatorname{div}\left(|\nabla u|^{p-2} \nabla u\right)=0 \text { in } \bar{\Omega}^{c}, \\
u=1 \text { on } \partial \Omega, \\
u(x) \rightarrow 0 \text { as }|x| \rightarrow \infty .
\end{array}\right.
$$

Pólya-Szegö principle yields the well-known isocapacitary inequality, telling that, among all sets with given volume, balls have the smallest possible $p$-capacity, namely

$$
\operatorname{cap}_{p}(\Omega)-\operatorname{cap}_{p}\left(B_{r}\right) \geq 0 .
$$

Here $r$ is such that $\left|B_{r}\right|=|\Omega|$, where $|\cdot|$ denotes the Lebesgue measure.

Indeed, to prove (1.2) recall the notion of Schwarz symmetrization. Let $\Omega$ be an open set and let $u$ be its capacitary potential. Schwarz symmetrization provides us with a radially symmetric function $u^{*}$ such that, for every $t \in \mathbb{R}$,

$$
|\{x: u(x)>t\}|=\left|\left\{x: u^{*}(x)>t\right\}\right| .
$$

We use $u^{*}$ as a test function for the set $\left\{x: u^{*}(x)=1\right\}=B_{r}$ and we note that (1.3) yields that $\left|B_{r}\right|=|\Omega|$. Hence

$$
\operatorname{cap}_{p}\left(B_{r}\right) \leq \int_{\mathbb{R}^{N}}\left|\nabla u^{*}\right|^{p} d x \leq \int_{\mathbb{R}^{N}}|\nabla u|^{p} d x=\operatorname{cap}_{p}(\Omega) \quad|\Omega|=\left|B_{r}\right|,
$$

\footnotetext{
${ }^{1}$ Here $D^{1, p}$ denotes homogeneous Sobolev space, i.e. $D^{1, p}=\overline{C_{c}^{\infty}\left(\mathbb{R}^{n}\right)} \|^{\|\cdot\|_{D^{1, p}}}$, where

$$
\|u\|_{D^{1, p}}:=\|\nabla u\|_{L^{p}\left(\mathbb{R}^{n}\right)}
$$
}


where the second inequality follows by Pólya-Szegö principle.

Inequality (1.2) is rigid, that is, equality is attained only when $\Omega$ coincides with a ball, up to a set of zero $p$-capacity. To see that, one may use that the equality in Pólya-Szegö inequality is attained only when $u$ is almost everywhere equal to a translate of $u^{*}$ (see, for example, [BZ88]).

It is natural to wonder whether this inequality is also stable, that is $\Omega \rightarrow B_{r}$, whenever $\operatorname{cap}_{p}(\Omega) \rightarrow \operatorname{cap}_{p}\left(B_{r}\right)$. This indeed turns out to be true and the right choice of the distance between sets is Fraenkel asymmetry, defined below.

Definition 1.1. Let $\Omega$ be an open set. The Fraenkel asymmetry of $\Omega, \mathcal{A}(\Omega)$, is defined as:

$$
\mathcal{A}(\Omega)=\inf \left\{\frac{|\Omega \Delta B|}{|B|}: B \text { is a ball with the same volume as } \Omega\right\} .
$$

To our knowledge, the first result of this sort goes back to [HHW91], where they considered the case of planar sets and $p=2^{2}$ and of convex sets in general dimension. The sharp stability inequality for $N=p=2$ has been given by Hansen and Nadirashvili in [HN92, Corollary 1]. For general dimension and $p$, the best result to our knowledge is due to Fusco, Maggi, and Pratelli in [FMP09] where they prove the following:

Theorem 1.2 ([FMP09]). There exists a constant $c=c(N, p)$ such that, for any open set $\Omega$

$$
\frac{\operatorname{cap}_{p}(\Omega)-\operatorname{cap}_{p}\left(B_{r}\right)}{r^{N-p}} \geq c \mathcal{A}(\Omega)^{2+p} .
$$

For the case $p=2$, the following inequality was obtained in a recent paper by De Philippis, Marini, and the author.

Theorem 1.3 ([DPMM19]). There exists a constant $c=c(N, p)$ such that, for any open set $\Omega$

$$
\frac{\operatorname{cap}_{2}(\Omega)-\operatorname{cap}_{2}\left(B_{r}\right)}{r^{N-2}} \geq c \mathcal{A}(\Omega)^{2} .
$$

The goal of this paper is to extend the result of Theorem 1.3 to the case of a general $p$.

1.2. Main result and strategy of the proof. The following is the main result of the paper. By the scaling $\operatorname{cap}_{p}(\lambda \Omega)=\lambda^{N-p} \operatorname{cap}_{p}(\Omega)$, we can also get the analogous result for $\Omega$ with arbitrary volume.

Theorem 1.4. Let $\Omega$ be an open set such that $|\Omega|=\left|B_{1}\right|$. Then there exists a constant $c=c(N, p)$ such that the following inequality holds:

$$
\operatorname{cap}_{p}(\Omega)-\operatorname{cap}_{p}\left(B_{1}\right) \geq c \mathcal{A}(\Omega)^{2} .
$$

\footnotetext{
${ }^{2}$ Note that for $N=p=2$ the infimum (1.1) is 0 and one has to use the notion of logarithmic capacity.
} 
Notice that by testing the inequality on ellipsoids, one can see that the exponent 2 on the right hand side of the inequality in Theorem 1.4 cannot be improved.

A natural way to tackle a problem like this is quantifying the proof of inequality (1.2). Indeed, that was done in [FMP09]. However, it seems that one is bound to get a non-sharp exponent on the right-hand side arguing in such a way. So instead we employ the approach of [CL12], devised for proving sharp isoperimetric inequality. The method, called Selection Principle, can be described as follows.

We first prove the theorem for smooth enough sets. This can be done by taking shape derivatives, writing Taylor expansion near the ball for $p$-capacity and providing relevant bounds for the second derivative and the remainder term. That is done in Section 2.

Then we prove the theorem for bounded sets of small asymmetry. We argue by contradiction. Suppose there exists a sequence of sets $\Omega_{j}$ such that $\frac{\operatorname{cap}_{p}\left(\Omega_{j}\right)-\operatorname{cap}_{p}\left(B_{1}\right)}{\mathcal{A}\left(\Omega_{j}\right)^{2}}$ converges to 0 . We then perturb the sequence $\left\{\Omega_{j}\right\}$ in such a way that the new sequence $\left\{\tilde{\Omega}_{j}\right\}$ still contradicts the theorem but also consists of minimizers of certain free boundary problems. By employing regularity results of [DP05], we infer that the sets $\tilde{\Omega}_{j}$ need to be smooth. That leads us to contradiction with the first step of the proof. This is the content of Sections 3-5.

Finally, in Sections 6 and 7 we reduce the general case to the one of bounded sets of small asymmetry. To reduce to the bounded sets, we provide bounds for the asymmetry and deficit of the set $\Omega \cap B_{R}$, where $\Omega$ is an arbitrary open set and $R$ is big enough. To reduce to the sets of small asymmetry we use the non-sharp quantitative inequality of [FMP09].

Note that as in [BDPV15] and [DPMM19], we replace the Fraenkel asymmetry (which roughly resembles a $L^{1}$ type norm) with a smoother (and stronger) version inspired by the distance among sets first used by Almgren, Taylor, and Wang in [ATW93] which resembles an $L^{2}$ type norm, see Section 2.3 for the exact definition.

Acknowledgements. The work of the author is supported by the INDAM-grant "Geometric Variational Problems" and by the H2020-MSCA-RISE-2017 PROJECT No. 778010 IPADEGAN. The author wishes to thank Guido De Philippis for many fruitful discussions.

\section{Fuglede's COMPUtATion}

In this section we are going to prove the validity of the quantitative isocapacitary inequality for sets close to the unit ball. More precisely, we are going to prove Theorem 1.4 for nearly spherical sets which are defined below. The proof is based on second variation argument as in [Fug89] (compare with [DPMM19, Section 2]).

Definition 2.1. An open bounded set $\Omega \subset \mathbb{R}^{N}$ is called nearly spherical of class $C^{2, \gamma}$ parametrized by $\varphi$, if there exists $\varphi \in C^{2, \gamma}$ with $\|\varphi\|_{L^{\infty}}<\frac{1}{2}$ such that

$$
\partial \Omega=\left\{(1+\varphi(x)) x: x \in \partial B_{1}\right\} .
$$

As mentioned in the introduction, we are going to work with another notion of asymmetry. Let us define it and state basic properties. 
Definition 2.2. Let $\Omega$ be an open set in $\mathbb{R}^{N}$. Then we define the asymmetry $\alpha$ in the following way:

$$
\alpha(\Omega)=\int_{\Omega \Delta B_{1}\left(x_{\Omega}\right)}|1-| x-x_{\Omega}|| d x .
$$

Here $x_{\Omega}$ denotes the barycenter of $\Omega$, namely $x_{\Omega}=f_{\Omega} x d x$.

Lemma 2.3 ([BDPV15, Lemma 4.2]). Let $\Omega \subset \mathbb{R}^{n}$, then

(i) There exists a constant $c=c(N)$ such that

$$
\alpha(\Omega) \geq c\left|\Omega \Delta B_{1}\left(x_{\Omega}\right)\right|^{2}
$$

for any open set $\Omega$.

(ii) There exists a constant $C=C(R)$ such that

$$
\left|\alpha\left(\Omega_{1}\right)-\alpha\left(\Omega_{2}\right)\right| \leq C\left|\Omega_{1} \Delta \Omega_{2}\right|
$$

for any $\Omega_{1}, \Omega_{2} \subset B_{R}$. In particular, if $1_{\Omega_{k}} \rightarrow 1_{\Omega}$ in $L^{1}\left(B_{R}\right)$ then $\alpha\left(\Omega_{k}\right) \rightarrow$ $\alpha(\Omega)$.

(iii) There exist constants $C=C(N), \delta=\delta(N)$ such that for every nearly spherical set $\Omega$ parametrized by $\varphi$ with $\|\varphi\|_{\infty} \leq \delta$ and $x_{\Omega}=0$

$$
\alpha(\Omega) \leq C\|\varphi\|_{L^{2}\left(\partial B_{1}\right)}^{2} .
$$

We are also going to use the following norm:

$$
\|\varphi\|_{H^{\frac{1}{2}\left(\partial B_{1}\right)}}^{2}:=\int_{\partial B_{1}} \varphi^{2} d \mathcal{H}^{N-1}+\int_{B_{1}^{c}}|\nabla H(\varphi)|^{2} d x
$$

where $H(\varphi)$ is the harmonic extension of $\varphi$ into $B^{c}$, i.e. it is the solution of

$$
\left\{\begin{array}{l}
\Delta H(\varphi)=0 \text { in } B_{1}^{c} \\
H(\varphi)=\varphi \text { on } \partial B_{1} \\
H(\varphi)(x) \rightarrow 0 \text { as }|x| \rightarrow \infty
\end{array}\right.
$$

in $D^{1,2}\left(\mathbb{R}^{N}\right)$. Note that this norm is equivalent to the standard one, where the second integral is replaced by Gagliardo seminorm (see for example [Gr, $(1,3,3,3)]$ ).

We now want to write Taylor expansion for $p$-capacity around the ball. Let us first introduce a technical lemma that is going to give us a sequence of sets along which we will be taking derivatives. One can find its proof in [BDPV15, Lemma A.1].

Lemma 2.4. Given $\gamma \in(0,1]$ there exists $\delta=\delta(N, \gamma)>0$ and a modulus of continuity $\omega$ such that for every nearly spherical set $\Omega$ parametrized by $\varphi$ with $\|\varphi\|_{C^{2, \gamma}\left(\partial B_{1}\right)}<\delta$ and $|\Omega|=\left|B_{1}\right|$, we can find an autonomous vector field $X_{\varphi}$ for which the following holds true:

(i) $\operatorname{div} X_{\varphi}=0$ in a $\delta$-neighborhood of $\partial B_{1}$;

(ii) if $\Phi_{t}:=\Phi(t, x)$ is the flow of $X_{\varphi}$, i.e.

$$
\partial_{t} \Phi_{t}=X_{\varphi}\left(\Phi_{t}\right), \quad \Phi_{0}(x)=x,
$$

then $\Phi_{1}\left(\partial B_{1}\right)=\partial \Omega$ and $\left|\Phi_{t}\left(B_{1}\right)\right|=\left|B_{1}\right|$ for all $t \in[0,1]$;

(iii) $\bullet\left\|\Phi_{t}-I d\right\|_{C^{2, \gamma}} \leq \omega\left(\|\varphi\|_{C^{2, \gamma}\left(\partial B_{1}\right)}\right)$ for every $t \in[0,1]$, 
- $\left\|\varphi-\left(X_{\varphi} \cdot \nu_{B_{1}}\right)\right\|_{H^{\frac{1}{2}\left(\partial B_{1}\right)}} \leq \omega\left(\|\varphi\|_{L^{\infty}\left(\partial B_{1}\right)}\right)\|\varphi\|_{H^{\frac{1}{2}\left(\partial B_{1}\right)}}$,

- $(X \cdot x) \circ \Phi_{t}-X \cdot \nu_{B_{1}}=\left(X \cdot \nu_{B_{1}}\right) \psi_{t}, x \in \partial B_{1}$, where $\left\|\psi_{t}\right\|_{C^{2, \gamma}\left(\partial B_{1}\right)} \leq \omega\left(\|\varphi\|_{C^{2, \gamma}\left(\partial B_{1}\right)}\right)$.

Remark 2.5. Here and in the sequel we will denote by $\nu_{\Omega}$ the inner unit normal.

Following [FZ16] (note that we will be citing the preprint rather than the published version [FZ17] as it has more details), we consider perturbed functionals to make the equation non-degenerate.

Definition 2.6. We define perturbed $p$-capacity as follows:

$$
\operatorname{cap}_{p, \kappa}(\Omega)=\inf _{u \in W^{1, p}\left(\mathbb{R}^{N}\right)}\left\{\int_{\Omega^{c}}\left(\left(\kappa^{2}+|\nabla u|^{2}\right)^{\frac{p}{2}}-\kappa^{p}\right) d x: u=1 \text { on } \Omega\right\} .
$$

Remark 2.7. Note that the infimum is achieved by the unique solution of the following equation

$$
\left\{\begin{array}{l}
\operatorname{div}\left(\left(\kappa^{2}+|\nabla u|^{2}\right)^{\frac{p-2}{2}} \nabla u\right)=0 \text { in } \Omega^{c} \\
u=1 \text { on } \partial \Omega \\
u(x) \rightarrow 0 \text { as }|x| \rightarrow \infty
\end{array}\right.
$$

We will denote the minimizer by $u_{\kappa, \Omega}$.

Let $\Phi_{t}$ be the flow from the lemma and define $\Omega_{t}=\Phi_{t}(B)$. For brevity we denote $u_{\kappa, t}:=u_{\kappa, \Omega_{t}}$.

Remark 2.8. The function $u_{\kappa, t}$ satisfies the following equation

$$
\left\{\begin{array}{l}
\operatorname{div}\left(\left(\kappa^{2}+\left|\nabla u_{\kappa, t}\right|^{2}\right)^{\frac{p-2}{2}} \nabla u_{\kappa, t}\right)=0 \text { in } \Omega_{t}^{c}, \\
u_{\kappa, t}=1 \text { on } \partial \Omega_{t} \\
u_{\kappa, t}(x) \rightarrow 0 \text { as }|x| \rightarrow \infty .
\end{array}\right.
$$

We also note that $\nabla u_{\kappa, t}=\left|\nabla u_{\kappa, t}\right| \nu_{\partial \Omega_{t}}$ on $\partial \Omega_{t}$ since it is constant on the boundary and less than 1 outside of the set by maximum principle.

We want to differentiate the perturbed $p$-capacity of $\Omega_{t}$ in $t$. We introduce the following notation

$$
c_{k}(t):=\int_{\Omega_{t}^{c}}\left(\left(\kappa^{2}+\left|\nabla u_{\kappa, t}\right|^{2}\right)^{\frac{p}{2}}-\kappa^{p}\right) d x .
$$

Since for any $\kappa>0$ equation (2.1) is elliptic, the following standard (see e.g. [SZ, Proposition 3.1]) differentiability result holds.

Lemma 2.9 (Shape derivative of $u_{\kappa, t}$ ). For any $\kappa>0$ the derivative of $u_{\kappa, t}$ in $t$ exists and it solves the following equation

$$
\left\{\begin{array}{l}
\operatorname{div}\left(\left(\kappa^{2}+\left|\nabla u_{\kappa, t}\right|^{2}\right)^{\frac{p-2}{2}} \nabla \dot{u}_{\kappa, t}\right. \\
\left.\quad+(p-2)\left(\kappa^{2}+\left|\nabla u_{\kappa, t}\right|^{2}\right)^{\frac{p-4}{2}}\left(\nabla u_{\kappa, t} \cdot \nabla \dot{u}_{\kappa, t}\right) \nabla u_{\kappa, t}\right)=0 \text { in } \Omega_{t}^{c}, \\
\dot{u}_{\kappa, t}=-\nabla u_{\kappa, t} \cdot X \text { on } \partial \Omega_{t} .
\end{array}\right.
$$


We want to see what happens near the ball for the initial functional. To that end, we compute $u_{0}:=u_{0,0}$ and its gradient:

$$
u_{0}=|x|^{\frac{p-n}{p-1}}, \nabla u_{0}=\frac{p-n}{p-1}|x|^{\frac{p-n}{p-1}-1} \theta
$$

where $\theta:=\frac{x}{|x|}$.

Theorem 2.10 (convergence of $\left.u_{\kappa, t}\right)$. Let $\kappa \in[0,1], p>1, \alpha \in(0,1), R>1$. There exist $\beta \in(0, \alpha)$ and a modulus of continuity $\omega=\omega(p, \alpha, n)$ such that if $\Omega$ is a $C^{2, \alpha}$ nearly spherical set parametrized by $\varphi$ and $\|\varphi\|_{C^{2, \alpha}(\partial B)}<\delta$, then for all $t \in[0,1]$ and $\kappa \in[0,1]$ we have

$$
\left\|u_{0}-u_{\kappa, t} \circ \Phi_{t}\right\|_{C^{1, \beta}\left(B_{R} \backslash B_{1}\right)} \leq \omega\left(\|\varphi\|_{C^{2, \alpha}(\partial B)}+\kappa\right) .
$$

Moreover, there exist $\delta^{\prime}>0,0<\gamma<\alpha$ and a modulus of continuity $\omega^{\prime}=$ $\omega^{\prime}(p, \alpha, n, \varepsilon)$, such that if $\|\varphi\|_{C^{2, \alpha}(\partial B)}+\kappa<\delta^{\prime}$, then for all $t \in[0,1]$

$$
\left\|u_{0}-u_{\kappa, t} \circ \Phi_{t}\right\|_{C^{2, \gamma}\left(B_{R} \backslash B_{1}\right)} \leq \omega^{\prime}\left(\|\varphi\|_{C^{2, \alpha}(\partial B)}+\kappa\right) .
$$

Proof. The proof goes in the same way as the one of [FZ16, Theorem 2.2]. We reproduce it here for the reader's convenience.

First, we notice that regularity for degenerate elliptic equations (see [L88, Theorem 1]) gives us

$$
\left\|u_{\kappa, t}\right\|_{C^{1, \beta^{\prime}}\left(B_{R} \backslash \Omega_{t}\right)} \leq C=C(p, \alpha, n, \delta)
$$

for some $\beta^{\prime} \in(0, \alpha)$, and every $\kappa \in[0,1], t \in[0,1]$. Fix $\beta \in\left(0, \beta^{\prime}\right)$. To prove the first inequality we argue by contradiction. Suppose there exist sequences $\left\{\varphi_{j}\right\},\left\{\kappa_{j}\right\}$, $\left\{t_{j}\right\}$ such that $\left\|\varphi_{j}\right\|_{C^{2, \alpha}(\partial B)}+\kappa_{j} \rightarrow 0$,

$$
\limsup _{j \rightarrow \infty}\left\|u_{0}-u_{\kappa_{j}, t_{j}} \circ \Phi_{t_{j}}^{j}\right\|_{C^{1, \beta}\left(B_{R} \backslash B_{1}\right)}>0,
$$

where $\Phi^{j}$ is the flow from Lemma 2.4 associated with $\varphi_{j}$. Using (2.3), we extract a (non-relabelled) subsequence such that $\tilde{u}_{j}:=u_{\kappa_{j}, t_{j}} \circ \Phi_{t_{j}}^{j}$ converges to a function $u$ in $C^{1, \beta}$. Each function $\tilde{u}_{j}$ satisfies

$$
\left\{\begin{array}{l}
\operatorname{div}\left(\left(\kappa_{j}^{2}+\left|\left(\left(\nabla \Phi_{t_{j}}^{j}\right)^{-1}\right)^{t} \nabla \tilde{u}_{j}\right|^{2}\right)^{\frac{p-2}{2}} M_{j} \nabla \tilde{u}_{j}\right)=0 \text { in } B^{c} \\
\tilde{u}_{j}=1 \text { on } \partial B \\
\tilde{u}_{j}(x) \rightarrow 0 \text { as }|x| \rightarrow \infty
\end{array}\right.
$$

where $M_{j}=\operatorname{det} \nabla \Phi_{t_{j}}^{j}\left(\nabla \Phi_{t_{j}}^{j}\right)^{-1}\left(\left(\nabla \Phi_{t_{j}}^{j}\right)^{-1}\right)^{t}$. Thus, $u$, as a limit of $\tilde{u}_{j}$ in $C^{1, \beta}$, satisfies

$$
\left\{\begin{array}{l}
\operatorname{div}\left(|\nabla u|^{p-2} \nabla u\right)=0 \text { in } B^{c} \\
u=1 \text { on } \partial B \\
u(x) \rightarrow 0 \text { as }|x| \rightarrow \infty
\end{array}\right.
$$

meaning that $u$ coincides with $u_{0}$, which contradicts (2.4). 
To get convergence in $C^{2, \gamma}$, we notice that $0<c \leq\left|\nabla u_{0}\right| \leq C$ in $B_{R} \backslash B_{1}$. The $C^{1, \beta^{\prime}}$ converges gives us that the same is true for $\nabla u_{\kappa, t}$ if $\|\varphi\|_{C^{2, \alpha}(\partial B)}+\kappa$ is small enough. From here equation for $u_{\kappa, t}$ and Schauder estimates give us

$$
\left\|u_{\kappa, t}\right\|_{C^{2, \gamma^{\prime}}\left(B_{R} \backslash \Omega_{t}\right)} \leq C=C(p, \alpha, n, \delta) .
$$

We now can argue in the same way as we $\operatorname{did}$ for $C^{1, \beta^{\prime}}$ convergence.

\subsection{First derivative.}

Proposition 2.11. For $\kappa>0, t \in[0,1]$ the perturbed p-capacity is differentiable in $t$ and the following formula holds

$$
\begin{aligned}
c_{\kappa}^{\prime}(t)=- & p \int_{\partial \Omega_{t}}\left|\nabla u_{\kappa, t}\right|^{2}\left(\kappa^{2}+\left|\nabla u_{\kappa, t}\right|^{2}\right)^{\frac{p-2}{2}}(X \cdot \nu) d \mathcal{H}^{N-1} \\
& +\int_{\partial \Omega_{t}}\left(\left(\kappa^{2}+\left|\nabla u_{\kappa, t}\right|^{2}\right)^{\frac{p}{2}}-\kappa^{p}\right)(X \cdot \nu) d \mathcal{H}^{N-1} \\
=- & p \int_{\Omega_{t}^{c}} \operatorname{div}\left(\left|\nabla u_{\kappa, t}\right|^{2}\left(\kappa^{2}+\left|\nabla u_{\kappa, t}\right|^{2}\right)^{\frac{p-2}{2}} X\right) d x \\
& +\int_{\Omega_{t}^{c}} \operatorname{div}\left(\left(\left(\kappa^{2}+\left|\nabla u_{\kappa, t}\right|^{2}\right)^{\frac{p}{2}}-\kappa^{p}\right) X\right) d x .
\end{aligned}
$$

Moreover, for every $t \in[0,1]$ we also have

$$
c_{0}^{\prime}(t)=-(p-1) \int_{\partial \Omega_{t}}\left|\nabla u_{0, t}\right|^{p}(X \cdot \nu) d \mathcal{H}^{N-1} .
$$

Proof. By Hadamard's formula (see [HP05, Chapter 5]),

$$
\begin{aligned}
c_{\kappa}^{\prime}(t)= & \int_{\Omega_{t}^{c}} p \nabla u_{\kappa, t} \cdot \nabla \dot{u}_{\kappa, t}\left(\kappa^{2}+\left|\nabla u_{\kappa, t}\right|^{2}\right)^{\frac{p-2}{2}} d x+\int_{\partial \Omega_{t}}\left(\left(\kappa^{2}+\left|\nabla u_{\kappa, t}\right|^{2}\right)^{\frac{p}{2}}-\kappa^{p}\right)(X \cdot \nu) d \mathcal{H}^{N-1} \\
= & \int_{\partial \Omega_{t}}\left(-\nabla u_{\kappa, t} \cdot X\right) p\left(\kappa^{2}+\left|\nabla u_{\kappa, t}\right|^{2}\right)^{\frac{p-2}{2}} \nabla u_{\kappa, t} \cdot \nu d \mathcal{H}^{N-1} \\
& \quad+\int_{\partial \Omega_{t}}\left(\left(\kappa^{2}+\left|\nabla u_{\kappa, t}\right|^{2}\right)^{\frac{p}{2}}-\kappa^{p}\right)(X \cdot \nu) d \mathcal{H}^{N-1},
\end{aligned}
$$

where for the second equality we used the equations (2.1) and (2.2). It remains to notice that $\nabla u_{\kappa, t}=\left|\nabla u_{\kappa, t}\right| \nu$ on $\partial \Omega_{t}$ as noted in Remark 2.8. This gives us the first equality of (2.5), whereas the second equality of (2.5) follows from divergence theorem.

The convergence established in Theorem 2.10 gives us (2.6).

2.2. Second derivative. To state the results for the second derivative we need to introduce the following weighted Sobolev space:

$$
D^{1,2}\left(B^{c}, \mu\right):=\left\{u \in H_{l o c}^{1}\left(B^{c}\right): \int_{B^{c}}|\nabla u|^{2} d \mu<\infty\right\},
$$

where $d \mu=|x|^{\left(\frac{p-n}{p-1}-1\right)(p-2)} d x$. We denote by $D_{0}^{1,2}\left(B^{c}, \mu\right)$ the corresponding space of functions with zero trace on $\partial B$. 
We also denote the mean curvature of a smooth set $\Omega$ by $\mathscr{H}_{\Omega}\left(\mathscr{H}_{\Omega}=\operatorname{div} \nu_{\Omega}\right.$, note that $\left.\mathscr{H}_{\Omega_{t}^{c}}=-\mathscr{H}_{\Omega_{t}}\right)$.

Proposition 2.12. We define $X_{\tau}:=X-(X \cdot \nu) \nu$. Then for $\kappa>0, t \in[0,1]$ the perturbed p-capacity is twice differentiable and the following formula holds:

$$
\begin{aligned}
\frac{1}{p} c_{\kappa}^{\prime \prime}(t) & =\int_{\partial \Omega_{t}}\left(\kappa^{2}+\left|\nabla u_{\kappa, t}\right|^{2}\right)^{\frac{p-2}{2}}\left(\nabla \dot{u}_{\kappa, t} \cdot \nu\right) \dot{u}_{\kappa, t} d \mathcal{H}^{N-1} \\
& +(p-2) \int_{\partial \Omega_{t}}\left(\kappa^{2}+\left|\nabla u_{\kappa, t}\right|^{2}\right)^{\frac{p-4}{2}}\left(\nabla \dot{u}_{\kappa, t} \cdot \nabla u_{\kappa, t}\right)\left(\nabla \dot{u}_{\kappa, t} \cdot \nu\right) \dot{u}_{\kappa, t} d \mathcal{H}^{N-1} \\
& -\int_{\partial \Omega_{t}}\left(\kappa^{2}+\left|\nabla u_{\kappa, t}\right|^{2}\right)^{\frac{p-2}{2}}\left(\nabla^{2} u_{\kappa, t}\left[\nabla u_{\kappa, t}\right] \cdot X_{\tau}\right)(X \cdot \nu) d \mathcal{H}^{N-1} \\
& -(p-2) \int_{\partial \Omega_{t}}\left(\kappa^{2}+\left|\nabla u_{\kappa, t}\right|^{2}\right)^{\frac{p-4}{2}}\left|\nabla u_{\kappa, t}\right|^{2}\left(\nabla^{2} u_{\kappa, t}\left[\nabla u_{\kappa, t}\right] \cdot X_{\tau}\right)(X \cdot \nu) d \mathcal{H}^{N-1} \\
& +\int_{\partial \Omega_{t}}\left|\nabla u_{\kappa, t}\right|^{2}(X \cdot \nu)^{2}\left(\kappa^{2}+\left|\nabla u_{\kappa, t}\right|^{2}\right)^{\frac{p-2}{2}} \mathscr{H}_{\Omega_{t}^{c}} d \mathcal{H}^{N-1} .
\end{aligned}
$$

Moreover,

$$
\begin{aligned}
\left(\frac{p-1}{N-p}\right)^{p-2} \frac{1}{p} c_{0}^{\prime \prime}(0) & =-(N-1) \int_{\partial B} \dot{u}_{0}^{2} d \mathcal{H}^{N-1} \\
& +\int_{B^{c}}|x|^{(p-2)\left(\frac{p-N}{p-1}-1\right)}\left(\left|\nabla \dot{u}_{0}\right|^{2}+(p-2)\left(\theta \cdot \nabla \dot{u}_{0}\right)^{2}\right) d x
\end{aligned}
$$

where $\dot{u}_{0}$ solves

$$
\left\{\begin{array}{l}
\operatorname{div}\left(|x|^{(p-2)\left(\frac{p-N}{p-1}-1\right)} \nabla \dot{u}_{0}+(p-2)|x|^{(p-2)\left(\frac{p-N}{p-1}-1\right)}\left(\theta \cdot \nabla \dot{u}_{0}\right) \theta\right)=0 \text { in } B^{c} \\
\dot{u}_{0}=\frac{N-p}{p-1} \theta \cdot X \text { on } \partial B
\end{array}\right.
$$

in $W^{1,2}\left(B^{c}, d \mu\right)$.

Proof. Computation. First we use Hadamard's formula to differentiate the second equality of (2.5). Using then the divergence theorem, we get

$$
\begin{aligned}
& c_{\kappa}^{\prime \prime}(t)=\int_{\partial \Omega_{t}} p\left(\kappa^{2}+\left|\nabla u_{\kappa, t}\right|^{2}\right)^{(p-2) / 2}\left(\nabla u_{\kappa, t} \cdot \nabla \dot{u}_{\kappa, t}\right)(X \cdot \nu) d \mathcal{H}^{N-1} \\
& +\int_{\partial \Omega_{t}} \operatorname{div}\left(\left(\left(\kappa^{2}+\left|\nabla u_{\kappa, t}\right|^{2}\right)^{\frac{p}{2}}-\kappa^{p}\right) X\right)(X \cdot \nu) d \mathcal{H}^{N-1} \\
& -p \int_{\partial \Omega_{t}}\left((p-2) \kappa^{2}+\left|\nabla u_{\kappa, t}\right|^{2}\right)^{(p-4) / 2}\left|\nabla u_{\kappa, t}\right|^{2}\left(\nabla u_{\kappa, t} \cdot \nabla \dot{u}_{\kappa, t}\right)(X \cdot \nu) d \mathcal{H}^{N-1} \\
& -2 p \int_{\partial \Omega_{t}} 2\left(\kappa^{2}+\left|\nabla u_{\kappa, t}\right|^{2}\right)^{(p-2) / 2}\left(\nabla u_{\kappa, t} \cdot \nabla \dot{u}_{\kappa, t}\right)(X \cdot \nu) d \mathcal{H}^{N-1} \\
& -p \int_{\partial \Omega_{t}} \operatorname{div}\left(\left(\kappa^{2}+\left|\nabla u_{\kappa, t}\right|^{2}\right)^{(p-2) / 2}\left|\nabla u_{\kappa, t}\right|^{2} X\right)(X \cdot \nu) d \mathcal{H}^{N-1} .
\end{aligned}
$$


Using (2.2), and the fact that $X$ is divergence-free, we obtain

$$
\begin{aligned}
& c_{\kappa}^{\prime \prime}(t)=p \int_{\partial \Omega_{t}}\left(\kappa^{2}+\left|\nabla u_{\kappa, t}\right|^{2}\right)^{(p-2) / 2} \dot{u}_{\kappa, t}\left(\nabla \dot{u}_{\kappa, t} \cdot \nu\right) d \mathcal{H}^{n-1} \\
& -\int_{\partial \Omega_{t}} p\left(\kappa^{2}+\left|\nabla u_{\kappa, t}\right|^{2}\right)^{(p-2) / 2}\left(\nabla^{2} u_{\kappa, t}\left[\nabla u_{\kappa, t}\right] \cdot X\right)(X \cdot \nu) d \mathcal{H}^{n-1} \\
& +p \int_{\partial \Omega_{t}}\left((p-2)\left(\kappa^{2}+\left|\nabla u_{\kappa, t}\right|^{2}\right)^{(p-4) / 2}\left|\nabla u_{\kappa, t}\right|^{2}\right) \dot{u}_{\kappa, t}\left(\nabla \dot{u}_{\kappa, t} \cdot \nu\right) \\
& -p(p-2) \int_{\partial \Omega_{t}}\left(\kappa^{2}+\left|\nabla u_{\kappa, t}\right|^{2}\right)^{(p-4) / 2}\left|\nabla u_{\kappa, t}\right|^{2}\left(\nabla^{2} u_{\kappa, t}\left[\nabla u_{\kappa, t}\right] \cdot X\right)(X \cdot \nu) d \mathcal{H}^{n-1} .
\end{aligned}
$$

We now use Remark 2.8 to get the following equality on the boundary

$$
\begin{aligned}
0 & =\operatorname{div}\left(\left(\kappa^{2}+\left|\nabla u_{\kappa, t}\right|^{2}\right)^{\frac{p-2}{2}} \nabla u_{\kappa, t}\right) \\
& =\left(\kappa^{2}+\left|\nabla u_{\kappa, t}\right|^{2}\right)^{\frac{p-2}{2}}\left|\nabla u_{\kappa, t}\right| \mathscr{H}_{\Omega_{t}^{c}}+\left(\kappa^{2}+\left|\nabla u_{\kappa, t}\right|^{2}\right)^{\frac{p-2}{2}} \nabla^{2} u_{\kappa, t}[\nu] \cdot \nu \\
& +(p-2)\left(\kappa^{2}+\left|\nabla u_{\kappa, t}\right|^{2}\right)^{\frac{p-4}{2}}\left|\nabla u_{\kappa, t}\right|^{2} \nabla^{2} u_{\kappa, t}[\nu] \cdot \nu .
\end{aligned}
$$

Now we plug (2.10) into (2.9) and get (2.7).

Convergence. Fix $R>1$. By Schauder estimates functions $u_{\kappa, t} \circ \Phi_{t}$ are equibounded in $C^{2, \gamma}\left(B_{R} \backslash B\right)$ and $\left|\nabla u_{\kappa, t}\right| \in(c(R), C(R))$ for $\kappa, t$ small. Thus, from (2.2), using classical elliptic estimates we get that $\dot{u}_{\kappa, t} \circ \Phi_{t}$ are equibounded in $C^{1, \gamma}\left(B_{R} \backslash B\right)$ and up to a subsequence converge to a function $\hat{w} \in C^{1}\left(B^{c}\right)$ uniformly on compacts.

Using $\dot{u}_{\kappa, t}$ as a test function in (2.2) and applying divergence theorem, we get

$$
\begin{aligned}
& \int_{\Omega_{t}^{c}}\left(\kappa^{2}+\left|\nabla u_{\kappa, t}\right|^{2}\right)^{\frac{p-2}{2}}\left|\nabla \dot{u}_{\kappa, t}\right|^{2} d x+(p-2) \int_{\Omega_{t}^{c}}\left(\kappa^{2}+\left|\nabla u_{\kappa, t}\right|^{2}\right)^{\frac{p-4}{2}}\left(\nabla u_{\kappa, t} \cdot \nabla \dot{u}_{\kappa, t}\right)^{2} d x \\
& =\int_{\partial \Omega_{t}^{c}}\left(\kappa^{2}+\left|\nabla u_{\kappa, t}\right|^{2}\right)^{\frac{p-2}{2}} \dot{u}_{\kappa, t} \nabla \dot{u}_{\kappa, t} \cdot \nu \\
& \quad+\int_{\partial \Omega_{t}^{c}}(p-2)\left(\kappa^{2}+\left|\nabla u_{\kappa, t}\right|^{2}\right)^{\frac{p-4}{2}}\left(\nabla u_{\kappa, t} \cdot \nabla \dot{u}_{\kappa, t}\right) \dot{u}_{\kappa, t} \nabla u_{\kappa, t} \cdot \nu \leq C .
\end{aligned}
$$

That means that $\hat{w} \in D^{1,2}\left(B^{c}, \mu\right)$. Passing to the limit in $(2.2)$ as $(\kappa, t) \rightarrow(0,0)$, we get

$$
\int_{B^{c}} \nabla \hat{w} \cdot \nabla v+(p-2)(\theta \cdot \nabla \hat{w})(\theta \cdot \nabla v) d \mu=0
$$

for any $v \in W^{1,2}\left(B_{R} \backslash \bar{B}\right)$ with compact support in $B_{R} \backslash \bar{B}$.

It remains to show that the same identity holds for every $v$ in $D_{0}^{1,2}\left(B^{c} ; \mu\right)$ and that $\hat{w}$ is the unique solution of the equation $(2.11)$ in $D^{1,2}\left(B^{c} ; \mu\right)$ with a proper boundary condition. To that end, we fix $R>1$ and a cut-off function $\eta_{R}$ such that $\eta_{R} \equiv 1$ in $B_{R} \backslash B, \eta_{R} \equiv 0$ in $B_{2 R}^{c}$ and $\left|\nabla \eta_{R}\right| \leq C / R$. We also fix a constant $c$ that we specify later, it will depend on $R$. For a function $v \in D_{0}^{1,2}\left(B^{c} ; \mu\right)$ we plug $(v-c) \eta_{R}$ 
into (2.11) to get

$$
\begin{aligned}
& \int_{B^{c}} \eta_{R} \nabla \hat{w} \cdot \nabla v+(p-2) \eta_{R}(\theta \cdot \nabla \hat{w})(\theta \cdot \nabla v) d \mu \\
& =\int_{B^{c}} \eta_{R} \nabla \hat{w} \cdot \nabla(v-c)+(p-2) \eta_{R}(\theta \cdot \nabla \hat{w})(\theta \cdot \nabla(v-c)) d \mu \\
& =-\left(\int_{B^{c}}(v-c) \nabla \hat{w} \cdot \nabla \eta_{R}+(p-2)(v-c)(\theta \cdot \nabla \hat{w})\left(\theta \cdot \nabla \eta_{R}\right) d \mu\right) .
\end{aligned}
$$

Claim: $\int_{B^{c}}(v-c) \nabla \hat{w} \cdot \nabla \eta_{R}+(p-2)(v-c)(\theta \cdot \nabla \hat{w})\left(\theta \cdot \nabla \eta_{R}\right) d \mu \rightarrow 0$ as $R \rightarrow \infty$. Indeed, we have

$$
\begin{aligned}
& \left|\int_{B^{c}}(v-c) \nabla \hat{w} \cdot \nabla \eta_{R}+(p-2)(v-c)(\theta \cdot \nabla \hat{w})\left(\theta \cdot \nabla \eta_{R}\right) d \mu\right| \\
& =\left.\left|\int_{B_{2 R} \backslash B_{R}}\left((v-c) \nabla \hat{w} \cdot \nabla \eta_{R}+(p-2)(v-c)(\theta \cdot \nabla \hat{w})\left(\theta \cdot \nabla \eta_{R}\right)\right)\right| x\right|^{\left(\frac{p-N}{p-1}-1\right)(p-2)} d x \mid \\
& \leq(p-1) \frac{C}{R} \int_{B_{2 R} \backslash B_{R}}|v-c||\nabla \hat{w}||x|^{\left(\frac{p-N}{p-1}-1\right)(p-2)} d x \\
& \leq \frac{C}{R}\left(\int_{B_{2 R} \backslash B_{R}}|\nabla \hat{w}|^{2}|x|^{\left(\frac{p-N}{p-1}-1\right)(p-2)} d x\right)^{1 / 2}\left(\int_{B_{2 R} \backslash B_{R}}|v-c|^{2}|x|^{\left(\frac{p-N}{p-1}-1\right)(p-2)} d x\right)^{1 / 2} .
\end{aligned}
$$

Since we know that $w \in D^{1,2}\left(B^{c} ; \mu\right)$, if we manage to show that

$$
\frac{1}{R}\left(\int_{B_{2 R} \backslash B_{R}}|v-c|^{2}|x|^{\left(\frac{p-N}{p-1}-1\right)(p-2)} d x\right)^{1 / 2} \leq C
$$

for some $C=C(N, p)$, the claim will be proven. For convenience we denote $\gamma:=$ $\left(\frac{p-N}{p-1}-1\right)(p-2)$. We now choose $c=f_{B_{2 R} \backslash B_{R}} v$ and use Poincaré inequality to get

$$
\begin{aligned}
\int_{B_{2 R} \backslash B_{R}}|v-c|^{2} d x=\int_{B_{2 R} \backslash B_{R}} \mid v & \left.f_{B_{2 R} \backslash B_{R}} v\right|^{2} d x \leq C R^{2} \int_{B_{2 R} \backslash B_{R}}|\nabla v|^{2} d x \\
& =C R^{2-\gamma} \int_{B_{2 R} \backslash B_{R}}|\nabla v|^{2} R^{\gamma} d x \leq C R^{2-\gamma}
\end{aligned}
$$

where in the last inequality we used that $v \in D^{1,2}\left(B^{c} ; \mu\right)$. This yields $(2.12)$, since

$$
\frac{1}{R}\left(\int_{B_{2 R} \backslash B_{R}}|v-c|^{2}|x|^{\gamma} d x\right)^{1 / 2} \leq C R^{-1+\gamma / 2}\left(\int_{B_{2 R} \backslash B_{R}}|v-c|^{2} d x\right)^{1 / 2} \leq C,
$$

where in the last inequality we applied (2.13). Thus we proved the claim and so (2.11) holds for every $v \in D_{0}^{1,2}\left(B^{c} ; \mu\right)$. The boundary condition is the same as in (2.8) since $\dot{u}_{\kappa, t} \circ \Phi_{t}$ are converging to $\hat{w}$ regularly on $\partial B$. So, $\hat{w}$ solves the Dirichlet problem (2.8) and by uniqueness the whole sequence $\dot{u}_{\kappa, t} \circ \Phi_{t}$ converges to $\hat{w}$.

Lemma 2.13. There exists a modulus of continuity $\omega$ such that

$$
\left|c_{\kappa}^{\prime \prime}(t)-c_{\kappa}^{\prime \prime}(0)\right| \leq \omega\left(\|\varphi\|_{C^{2, \alpha}}+\kappa\right)\|X \cdot \nu\|_{H^{1 / 2}(\partial B)}^{2} .
$$


Proof. By divergence theorem and (2.2), using change of variables we can rewrite the second derivative of the energy in the following way:

$$
\frac{1}{p} c_{\kappa}^{\prime \prime}(t)=I_{1}(t)+I_{2}(t)+I_{3}(t)
$$

where

$$
\begin{aligned}
& I_{1}(t):=\int_{B}\left(\left(\kappa^{2}+\left|\nabla u_{\kappa, t}\right|^{2}\right)^{\frac{p-2}{2}}\left|\nabla \dot{u}_{\kappa, t}\right|^{2}\right) \circ \Phi_{t} \operatorname{det} \nabla \Phi_{t} d x \\
& +(p-2) \int_{B}\left(\left(\kappa^{2}+\left|\nabla u_{\kappa, t}\right|^{2}\right)^{\frac{p-4}{2}}\left(\nabla \dot{u}_{\kappa, t} \cdot \nabla u_{\kappa, t}\right)^{2}\right) \circ \Phi_{t} \operatorname{det} \nabla \Phi_{t} d x, \\
& I_{2}(t):=-\int_{\partial B}\left(\left(\kappa^{2}+\left|\nabla u_{\kappa, t}\right|^{2}\right)^{\frac{p-2}{2}}\left(\nabla^{2} u_{\kappa, t}\left[\nabla u_{\kappa, t}\right] \cdot X_{\tau}\right)(X \cdot \nu)\right) \circ \Phi_{t} J^{\partial B} \Phi_{t} d \mathcal{H}^{N-1} \\
& -(p-2) \int_{\partial B}\left(\left(\kappa^{2}+\left|\nabla u_{\kappa, t}\right|^{2}\right)^{\frac{p-4}{2}}\left|\nabla u_{\kappa, t}\right|^{2}\left(\nabla^{2} u_{\kappa, t}\left[\nabla u_{\kappa, t}\right] \cdot X_{\tau}\right)(X \cdot \nu)\right) \circ \Phi_{t} J^{\partial B} \Phi_{t} d \mathcal{H}^{N-1}, \\
& I_{3}(t):=\int_{\partial B}\left(\left|\nabla u_{\kappa, t}\right|^{2}(X \cdot \nu)^{2}\left(\kappa^{2}+\left|\nabla u_{\kappa, t}\right|^{2}\right)^{\frac{p-2}{2}} \mathscr{H}_{\Omega_{t}^{c}}\right) \circ \Phi_{t} J^{\partial B} \Phi_{t} d \mathcal{H}^{N-1} .
\end{aligned}
$$

By Lemma 2.4, we have

$\left\|\mathscr{H}_{\partial \Omega_{t}} \circ \Phi_{t}-\mathscr{H}_{\partial B}\right\|_{L^{\infty}(\partial B)}+\left\|J_{n-1} \Phi_{t}-1\right\|_{L^{\infty}(\partial B)}+\left\|\operatorname{det} \nabla \Phi_{t}-1\right\|_{L^{\infty}(\partial B)} \leq \omega\left(\|\varphi\|_{C^{2, \alpha}}\right)$.

In addition, by Lemma $2.4, X$ is parallel to $\theta$ in a neighborhood of $\partial B$, so we have

$$
\left|\left(X \cdot \nu_{\Omega_{t}}\right) \circ \Phi_{t}-X \cdot \nu_{B}\right| \leq \omega\left(\|\varphi\|_{C^{2, \alpha}}\right)\left|X \cdot \nu_{B}\right|
$$

as well as

$$
\left|X_{\tau} \circ \Phi_{t}\right| \leq \omega\left(\|\varphi\|_{C^{2, \alpha}}\right)\left|X \cdot \nu_{B}\right| .
$$

Thus, using Theorem 2.10 and noticing that $I_{2}(0)=0$, we get

$$
\left|I_{2}(t)-I_{2}(0)\right|+\left|I_{3}(t)-I_{3}(0)\right| \leq \omega\left(\|\varphi\|_{C^{2, \alpha}}+\kappa\right)\left\|X \cdot \nu_{B}\right\|_{L^{2}(\partial B)}^{2} .
$$

It remains to show that

$$
\left|I_{1}(t)-I_{1}(0)\right| \leq \omega\left(\|\varphi\|_{C^{2, \alpha}}+\kappa\right)\|X \cdot \nu\|_{H^{1 / 2}(\partial B)}^{2} .
$$

We are going to sketch the proof of (2.14), for more details see the proof of [FZ16, Lemma 2.7]. We first move the equation for $\dot{u}_{\kappa, t}$ onto the unit ball $B$. To that end, we denote $w_{\kappa, t}:=\dot{u}_{\kappa, t} \circ \Phi_{t}, \tilde{u}_{\kappa, t}:=u_{\kappa, t} \circ \Phi_{t}$, and $N_{t}=\left(\nabla \Phi_{t}\right)^{-1}\left(\left(\nabla \Phi_{t}\right)^{-1}\right)^{t}$. Then $w_{\kappa, t}$ satisfies

$$
\left\{\begin{array}{l}
\operatorname{div}\left(\left(\kappa^{2}+\left|\left(\left(\nabla \Phi_{t}\right)^{-1}\right)^{t} \nabla \tilde{u}_{\kappa, t}\right|^{2}\right)^{\frac{p-2}{2}} \operatorname{det} \nabla \Phi_{t} N_{t} \nabla w_{\kappa, t}\right. \\
\left.+(p-2)\left(\kappa^{2}+\left|\left(\left(\nabla \Phi_{t}\right)^{-1}\right)^{t} \nabla \tilde{u}_{\kappa, t}\right|^{2}\right)^{\frac{p-4}{2}} \operatorname{det} \nabla \Phi_{t}\left(N_{t} \nabla \tilde{u}_{\kappa, t} \cdot \nabla w_{\kappa, t}\right) N_{t} \nabla w_{\kappa, t}\right)=0 \text { in } B^{c}, \\
w_{\kappa, t}=-\left(\nabla u_{\kappa, t} \cdot X\right) \circ \Phi_{t} \text { on } \partial B
\end{array}\right.
$$

and

$$
\begin{aligned}
I_{1}(t) & :=\int_{B}\left(\kappa^{2}+\left|\left(\left(\nabla \Phi_{t}\right)^{-1}\right)^{t} \nabla \tilde{u}_{\kappa, t}\right|^{2}\right)^{\frac{p-2}{2}} N_{t} \nabla w_{\kappa, t} \cdot \nabla w_{\kappa, t} \operatorname{det} \nabla \Phi_{t} d x \\
& +(p-2) \int_{B}\left(\kappa^{2}+\left|\left(\left(\nabla \Phi_{t}\right)^{-1}\right)^{t} \nabla \tilde{u}_{\kappa, t}\right|^{2}\right)^{\frac{p-4}{2}}\left(N_{t} \nabla \tilde{u}_{\kappa, t} \cdot \nabla w_{\kappa, t}\right)^{2} \operatorname{det} \nabla \Phi_{t} d x .
\end{aligned}
$$


For convenience we define a bilinear form $L_{\kappa, t, \varphi}$ as

$$
\begin{aligned}
& L_{\kappa, t, \varphi}(u, v):=\int_{B}\left(\kappa^{2}+\left|\left(\left(\nabla \Phi_{t, \varphi}\right)^{-1}\right)^{t} \nabla \tilde{u}_{\kappa, t, \varphi}\right|^{2}\right)^{\frac{p-2}{2}} N_{t, \varphi} \nabla u \cdot \nabla v \operatorname{det} \nabla \Phi_{t} d x \\
& +(p-2) \int_{B}\left(\kappa^{2}+\left|\left(\left(\nabla \Phi_{t, \varphi}\right)^{-1}\right)^{t} \nabla \tilde{u}_{\kappa, t, \varphi}\right|^{2}\right)^{\frac{p-4}{2}}\left(N_{t} \nabla \tilde{u}_{\kappa, t} \cdot \nabla u\right)\left(N_{t} \nabla \tilde{u}_{\kappa, t} \cdot \nabla v\right) \operatorname{det} \nabla \Phi_{t} d x,
\end{aligned}
$$

so that proving (2.14) amounts to showing that

$$
\left|L_{\kappa, t, \varphi}\left(w_{\kappa, t, \varphi, \kappa, t, \varphi}\right)-L_{\kappa, 0, \varphi}\left(w_{\kappa, 0, \varphi, \kappa, t, \varphi}\right)\right| \leq \omega\left(\|\varphi\|_{C^{2, \alpha}}+\kappa\right)\|X \cdot \nu\|_{H^{1 / 2}(\partial B)}^{2} .
$$

We argue by contradiction. Assume there exist sequences $\kappa_{j} \rightarrow 0, t_{j} \rightarrow t \in[0,1]$, $\varphi_{j} \rightarrow 0$ in $C^{2, \alpha}(\partial B)$ such that

$$
\lim _{j \rightarrow \infty} \frac{L_{\kappa_{j}, t_{j}, \varphi_{j}}\left(w_{\kappa_{j}, t_{j}, \varphi_{j}}, w_{\kappa_{j}, t_{j}, \varphi_{j}}\right)}{\left\|X_{j} \cdot \nu_{B}\right\|_{H^{1 / 2}(\partial B)}^{2}} \neq \lim _{j \rightarrow \infty} \frac{L_{\kappa_{j}, 0, \varphi_{j}}\left(w_{\kappa_{j}, 0, \varphi_{j}}, w_{\kappa_{j}, 0, \varphi_{j}}\right)}{\left\|X_{j} \cdot \nu_{B}\right\|_{H^{1 / 2}(\partial B)}^{2}} .
$$

Note that we can assume that both limits are finite. We define

$$
\tilde{w}_{j}:=\frac{w_{\kappa_{j}, t_{j}, \varphi_{j}}}{\left\|X_{j} \cdot \nu_{B}\right\|_{H^{1 / 2}(\partial B)}}, \quad \tilde{w}_{0, j}:=\frac{w_{\kappa_{j}, 0, \varphi_{j}}}{\left\|X_{j} \cdot \nu_{B}\right\|_{H^{1 / 2}(\partial B)}} .
$$

One can easily show that $\tilde{w}_{j}-\tilde{w}_{0, j} \rightarrow 0$ strongly in $H^{1 / 2}(\partial B)$. A bit more work is required to show that $\tilde{w}_{j}-\tilde{w}_{0, j} \rightarrow 0$ strongly in $W^{1,2}\left(B_{R} \backslash B\right)$ for every $r \in(0,1)$. To do that, one can prove first that both $\tilde{w}_{j}$ and $\tilde{w}_{0, j}$ converge weakly to the unique solution in $D^{1,2}\left(B^{c}, \mu\right)$ of

$$
\left\{\begin{array}{l}
\operatorname{div}\left(|x|^{\frac{p-2}{p-1}} \nabla w+(p-2)|x|^{\frac{p-2}{p-1}}(\theta \cdot \nabla w) \theta\right)=0 \text { in } B^{c} \\
w=f \text { on } \partial B
\end{array}\right.
$$

where $f$ is the weak limit in $H^{1 / 2}(\partial B)$ of the restriction of $\tilde{w}_{j}$ on $\partial B$ (remember that the limit of restriction of $\tilde{w}_{0, j}$ is the same). To show the strong convergence consider $z_{j}$ - the harmonic extension of $\tilde{w}_{j}-\tilde{w}_{0, j}$ from $\partial B$ to $B^{c}$. Note that $z_{j}$ converges strongly to zero in $D^{1,2}\left(B^{c}\right)$. Denote by $\zeta \in C_{0}^{\infty}\left(B_{R}\right)$ a cut-off function such that $\zeta \equiv 1$ on $B_{R} \backslash B, 0 \leq \zeta \leq 1$. By divergence theorem we get

$$
\begin{aligned}
& L_{\kappa_{j}, t_{j}, \varphi_{j}}\left(\tilde{w}_{j}-\tilde{w}_{0, j},\left(\tilde{w}_{j}-\tilde{w}_{0, j}\right) \zeta\right)=L_{\kappa_{j}, t_{j}, \varphi_{j}}\left(\tilde{w}_{j}, z_{j} \zeta\right) \\
& -\left(L_{\kappa_{j}, t_{j}, \varphi_{j}}-L_{\kappa_{j}, 0, \varphi_{j}}\right)\left(\tilde{w}_{0, j},\left(\tilde{w}_{j}-\tilde{w}_{0, j}\right) \zeta\right)-L_{\kappa_{j}, 0, \varphi_{j}}\left(\tilde{w}_{0, j}, z_{j} \zeta\right) \rightarrow 0,
\end{aligned}
$$

which yields strong convergence of $\tilde{w}_{j}-\tilde{w}_{0, j}$ to zero in $W^{1,2}\left(B_{R} \backslash B\right)$. Finally, one can now show that

$$
\lim _{j \rightarrow \infty}\left(L_{\kappa_{j}, t_{j}, \varphi_{j}}\left(\tilde{w}_{j}, \tilde{w}_{j}\right)-L_{\kappa_{j}, 0, \varphi_{j}}\left(\tilde{w}_{0, j}, \tilde{w}_{0, j}\right)\right)=0
$$

contradicting (2.15).

Lemma 2.14. Given $\gamma \in(0,1]$, there exists $\delta=\delta(N, \gamma)>0$ and a modulus of continuity $\omega$ such that for every nearly spherical set $\Omega$ parametrized by $\varphi$ with $\|\varphi\|_{C^{2, \gamma}\left(\partial B_{1}\right)}<\delta$ and $|\Omega|=\left|B_{1}\right|$, we have

$$
\operatorname{cap}_{p}(\Omega) \geq \operatorname{cap}_{p}\left(B_{1}\right)+\frac{1}{2} \partial^{2} \operatorname{cap}_{p}\left(B_{1}\right)[\varphi, \varphi]-\omega\left(\|\varphi\|_{C^{2, \gamma}}\right)\|\varphi\|_{H^{\frac{1}{2}}\left(\partial B_{1}\right)}^{2},
$$


where

$$
\begin{aligned}
\left(\frac{p-1}{N-p}\right)^{p-2} & \frac{1}{p} \partial^{2} \operatorname{cap}_{p}\left(B_{1}\right)[\varphi, \varphi]:=-(N-1) \int_{\partial B}\left(\frac{N-p}{p-1} \varphi\right)^{2} d \mathcal{H}^{N-1} \\
+ & \int_{B^{c}}|x|^{(p-2)\left(\frac{p-N}{p-1}-1\right)}\left(|\nabla f(\varphi)|^{2}+(p-2)(\theta \cdot \nabla f(\varphi))^{2}\right) d x
\end{aligned}
$$

with $f(\varphi)$ satisfying

$$
\left\{\begin{array}{l}
\operatorname{div}\left(|x|^{(p-2)\left(\frac{p-N}{p-1}-1\right)} \nabla f(\varphi)+(p-2)|x|^{(p-2)\left(\frac{p-N}{p-1}-1\right)}(\theta \cdot \nabla f(\varphi)) \theta\right)=0 \text { in } B^{c}, \\
f(\varphi)=\frac{N-p}{p-1} \varphi \text { on } \partial B .
\end{array}\right.
$$

Proof. We write Taylor expansion for $c_{\kappa}$ :

$$
c_{\kappa}(1)=c_{\kappa}(0)+c_{\kappa}^{\prime}(0)+\frac{1}{2} c_{\kappa}^{\prime \prime}(0)+\frac{1}{2} \int_{0}^{1}(1-t)\left(c_{\kappa}^{\prime \prime}(t)-c_{\kappa}^{\prime \prime}(0)\right) d t .
$$

From isocapacitary inequality we know that $c_{0}^{\prime}(0)=0$. So, we get the desired inequality using Lemma 2.13 and passing to the limit as $\kappa \rightarrow 0$.

2.3. Inequality for nearly spherical sets. We now establish a quantitative inequality for nearly spherical sets in the spirit of those established by Fuglede in [Fug89], compare with [FZ16, Section 2].

Theorem 2.15. There exists $\delta=\delta(N), c=c(N)$ such that if $\Omega$ is a nearly spherical set of class $C^{2, \gamma}$ parametrized by $\varphi$ with $\|\varphi\|_{C^{2, \gamma}} \leq \delta,|\Omega|=\left|B_{1}\right|$ and $x_{\Omega}=0$, then

$$
\operatorname{cap}_{p}(\Omega)-\operatorname{cap}_{p}\left(B_{1}\right) \geq c\|\varphi\|_{H^{\frac{1}{2}\left(\partial B_{1}\right)}}^{2} .
$$

Remark 2.16. Note that by Lemma 2.3 this theorem gives us Theorem 1.4 for nearly spherical sets.

Proof. Let $X$ be the vector field from Lemma 2.4. We introduce the following notation:

$$
\hat{u}=-\frac{p-1}{p-N} \dot{u}_{0}, \Psi=\theta \cdot X .
$$

Then by Proposition $2.12 \hat{u}$ solves

$$
\left\{\begin{array}{l}
\operatorname{div}\left(|x|^{(p-2)\left(\frac{p-N}{p-1}-1\right)} \nabla \hat{u}+(p-2)|x|^{(p-2)\left(\frac{p-N}{p-1}-1\right)}(\theta \cdot \nabla \hat{u}) \theta\right)=0 \text { in } B^{c}, \\
\hat{u}=\Psi \text { on } \partial B
\end{array}\right.
$$

and

$$
\begin{aligned}
\left(\frac{p-1}{p-N}\right)^{p} \frac{1}{p} c_{0}^{\prime \prime}(0) & =-(N-1) \int_{\partial B} \hat{u}^{2} d \mathcal{H}^{N-1} \\
& +\int_{B^{c}}|x|^{(p-2)\left(\frac{p-N}{p-1}-1\right)}\left(|\nabla \hat{u}|^{2}+(p-2)(\theta \cdot \nabla \hat{u})^{2}\right) d x .
\end{aligned}
$$

We introduce the following notation:

$$
\begin{aligned}
Q[\Psi] & =-(N-1) \int_{\partial B} \hat{u}^{2} d \mathcal{H}^{N-1} \\
& +\int_{B^{c}}|x|^{(p-2)\left(\frac{p-N}{p-1}-1\right)}\left(|\nabla \hat{u}|^{2}+(p-2)(\theta \cdot \nabla \hat{u})^{2}\right) d x .
\end{aligned}
$$


We write $\Psi$ in the basis of spherical harmonics, i.e.

$$
\Psi=\sum_{k=0}^{\infty} \sum_{i=1}^{M(k, N)} a_{k, i} Y_{k, i}
$$

where $Y_{k, i}$ for $i=1, \ldots, M(k, N)$ are harmonic polynomials of degree $k$, normalized so that $\left\|Y_{k, i}\right\|_{L^{2}(\partial B)}=1$. By Lemma 2.4 we have that $\|\Psi\|_{H^{1 / 2}\left(\partial B_{1}\right)} \geq c\|\varphi\|_{H^{1 / 2}\left(\partial B_{1}\right)}$ if $\delta$ is small enough. Thus, to prove the theorem, by Lemma 2.14 it is enough to show that $Q[\Psi] \geq c\|\Psi\|_{H^{1 / 2}\left(\partial B_{1}\right)}^{2}$, or, equivalently, that

$$
Q[\Psi]=\sum_{k=0}^{\infty} \sum_{i=1}^{M(k, N)} a_{k, i}^{2} Q\left[Y_{k, i}\right] \geq c \sum_{k=0}^{\infty} \sum_{i=1}^{M(k, N)}(k+1) a_{k, i}^{2}
$$

We first note $\int_{\partial B} \Psi=0$ as $\Phi_{t}$ conserves volume, and thus $a_{0}=0$. We then bound $\sum_{i=1}^{n} a_{1, i}^{2}$. We recall that $x_{\Omega}=0$, hence

$$
\int_{\partial B} x\left((1+\varphi)^{N+1}-1\right) d \mathcal{H}^{N-1}=0
$$

and consequently, for any $\varepsilon>0$ if $\delta$ is small enough we get

$$
\left|\int_{\partial B} x \varphi d \mathcal{H}^{N-1}\right| \leq \varepsilon\|\varphi\|_{L^{2}(\partial B)}
$$

By Lemma 2.4 this in turn yields

$$
\left|\int_{\partial B} x \Psi d \mathcal{H}^{N-1}\right| \leq 2 \varepsilon\|\Psi\|_{L^{2}(\partial B)}
$$

if $\delta$ is small enough. So we get

$$
\sum_{i=1}^{n} a_{1, i}^{2} \leq 2 \sum_{k=2}^{\infty} \sum_{i=1}^{M(k, N)}(k+1) a_{k, i}^{2}
$$

for $\delta$ small enough and to prove (2.16) it remains to show that

$$
\sum_{k=0}^{\infty} \sum_{i=1}^{M(k, N)} a_{k, i}^{2} Q\left[Y_{k, i}\right] \geq c \sum_{k=2}^{\infty} \sum_{i=1}^{M(k, N)}(k+1) a_{k, i}^{2} .
$$

We denote by $u_{k, i}$ the function $\hat{u}$ corresponding to $Y_{k, i}$ on the boundary. Then a straightforward computation tells us that

$$
u_{k, i}=|x|^{\alpha_{k}} Y_{k, i},
$$

where $\alpha_{k}<0$ is the only negative solution of the following quadratic equation:

$$
(p-1) \alpha_{k}^{2}+(N-p) \alpha_{k}-k(k+N-2)=0 .
$$

Remembering that

$$
\int_{\partial B}\left|Y_{k, i}\right|^{2} d \mathcal{H}^{N-1}=1, \int_{\partial B}\left|\nabla_{\tau} Y_{k, i}\right|^{2} d \mathcal{H}^{N-1}=k(k+N-2),
$$


we get that

$$
\begin{array}{r}
Q\left[Y_{k, i}\right]=-(N-1)-\frac{\alpha_{k}^{2}(p-1)+k(k+N-2)}{(p-2)\left(\frac{p-N}{p-1}-1\right)+2\left(\alpha_{k}-1\right)+N} \\
=-(N-1)-\frac{\left(2 k(k+N-2)-(N-p) \alpha_{k}\right)(p-1)}{(p-1) 2 \alpha_{k}+N-p},
\end{array}
$$

where we used (2.18). Now, since by (2.18) we have

$$
\alpha_{k}=-\frac{N-p+\sqrt{(N-p)^{2}+4(p-1) k(k+N-2)}}{2(p-1)},
$$

we get after straightforward computations

$$
Q\left[Y_{k, i}\right]=-(N-1)+\frac{N-p+\sqrt{(N-p)^{2}+4(p-1) k(k+N-2)}}{2} .
$$

Notice that

$$
Q\left[Y_{k, i}\right] \geq c k \text { for } k \geq 2
$$

for some $c=c(N, p)>0$. This gives us (2.17) and hence (2.16) and so we conclude the proof of the theorem.

\section{STABILITy FOR BOUNDED SETS With SMALL ASYMMETRY}

This section, together with the two subsequent ones, is dedicated to the proof of the following theorem.

Theorem 3.1. There exist constants $c=c(N, R), \varepsilon_{0}=\varepsilon_{0}(N, R)$ such that for any open set $\Omega \subset B_{R}$ with $|\Omega|=\left|B_{1}\right|$ and $\alpha(\Omega) \leq \varepsilon_{0}$ the following inequality holds:

$$
\operatorname{cap}_{p}(\Omega)-\operatorname{cap}_{p}\left(B_{1}\right) \geq c \alpha(\Omega) .
$$

To prove Theorem 3.1 we are going to argue by contradiction. Suppose that the theorem doesn't hold. Then there exists a sequence of open sets $\tilde{\Omega}_{j} \subset B_{R}$ such that

$$
\left|\tilde{\Omega}_{j}\right|=\left|B_{1}\right|, \quad \alpha\left(\tilde{\Omega}_{j}\right)=\varepsilon_{j} \rightarrow 0, \quad \frac{\operatorname{cap}_{p}\left(\tilde{\Omega}_{j}\right)-\operatorname{cap}_{p}\left(B_{1}\right)}{\varepsilon_{j}} \leq \sigma^{4}
$$

for some small $\sigma$ to be chosen later. We then perturb the sequence $\tilde{\Omega}_{j}$ so that it converges to $B_{1}$ in a smooth way. More precisely, we are going to show the following.

Theorem 3.2 (Selection Principle). There exists $\tilde{\sigma}=\tilde{\sigma}(N, R)$ such that if one has a contradicting sequence $\tilde{\Omega}_{j}$ as the one described above in (3.1) with $\sigma<\tilde{\sigma}$, then there exists a sequence of smooth open sets $U_{j}$ such that

(i) $\left|U_{j}\right|=\left|B_{1}\right|$,

(ii) $\partial U_{j} \rightarrow \partial B_{1}$ in $C^{k}$ for every $k$,

(iii) $\limsup _{j \rightarrow \infty} \frac{\operatorname{cap}_{p}\left(U_{j}\right)-\operatorname{cap}_{p}\left(B_{1}\right)}{\alpha\left(\Omega_{j}\right)} \leq C \sigma$ for some $C=C(N, R)$ constant,

(iv) the barycenter of every $\Omega_{j}$ is in the origin. 
Proof of Theorem 3.1 assuming Selection Principle. Suppose Theorem 3.1 does not hold. Then for any $\sigma>0$ we can find a contradicting sequence $\tilde{\Omega}_{j}$ as in (3.1). We apply Theorem 3.2 to $\tilde{\Omega}_{j}$ to get a smooth contradicting sequence $U_{j}$.

By the properties of $U_{j}$, we have that for $j$ big enough $U_{j}$ is a nearly spherical set with barycenter at the origin and with volume $\left|B_{1}\right|$. Thus, we can use Theorem 2.15 and get

$$
c(N, R) \leq \limsup _{j \rightarrow \infty} \frac{\operatorname{cap}_{*}\left(U_{j}\right)-\operatorname{cap}_{*}\left(B_{1}\right)}{\alpha_{*}\left(\Omega_{j}\right)} \leq C(N, R) \sigma .
$$

But this cannot happen for $\sigma=\sigma(N, R)$ small enough.

\section{Proof of Theorem 3.2: Existence and first properties}

4.1. Getting rid of the volume constraint. The first step consists in getting rid of the volume constraint in the isocapacitary inequality. Note that this has to be done locally since, by scaling, globally there exists no Lagrange multiplier. Furthermore, to apply the regularity theory for free boundary problems, it is crucial to introduce a monotone dependence on the volume. To this end, let us set, following [AAC86],

$$
f_{\eta}(s):= \begin{cases}-\frac{1}{\eta}\left(s-\omega_{N}\right), & s \leq \omega_{N} \\ -\eta\left(s-\omega_{N}\right), & s \geq \omega_{N}\end{cases}
$$

and let us consider the new functional

$$
\mathscr{C}_{\eta}(\Omega)=\operatorname{cap}_{p}(\Omega)+f_{\eta}(|\Omega|) .
$$

We now show that the above functional is uniquely minimized by balls. Note also that $f_{\eta}$ satisfies

$$
\eta(t-s) \leq f_{\eta}(s)-f_{\eta}(t) \leq \frac{(t-s)}{\eta} \quad \text { for all } 0 \leq s \leq t .
$$

Lemma 4.1. There exists an $\hat{\eta}=\hat{\eta}(R)>0$ such that the only minimizer of $\mathscr{C}_{\hat{\eta}}$ in the class of sets contained in $B_{R}$ is a translate of the unit unit ball $B_{1}$.

Moreover, there exists $c=c(R)>0$ such that for any ball $B_{r}$ with $0<r<R$, one has

$$
\mathscr{C}_{\hat{\eta}}\left(B_{r}\right)-\mathscr{C}_{\hat{\eta}}\left(B_{1}\right) \geq c|r-1| .
$$

Proof. Suppose that $\Omega$ is a minimizer of $\mathscr{C}_{\hat{\eta}}$ in the class of sets contained in $B_{R}$. Let $r$ be such that $\left|B_{r}\right|=|\Omega|$. Then, by symmetrization

$$
\mathscr{C}_{\hat{\eta}}(\Omega)=\operatorname{cap}_{p}(\Omega)+f_{\hat{\eta}}(|\Omega|) \geq \operatorname{cap}_{p}\left(B_{r}\right)+f_{\hat{\eta}}(|\Omega|)=\mathscr{C}_{\hat{\eta}}\left(B_{r}\right) .
$$

Moreover, the equality holds only if $\Omega$ is a translation of the ball $B_{r}$. So, any minimizer should be a translate of a ball of a radius $r \in(0, R]$, and we need to prove that a function $g:(0, R] \rightarrow \mathbb{R}_{+}$defined as

$$
g(r):=\operatorname{cap}_{p}\left(B_{r}\right)+f_{\hat{\eta}}\left(\left|B_{r}\right|\right)=\operatorname{cap}\left(B_{1}\right) r^{n-p}+f_{\hat{\eta}}\left(\left|B_{r}\right|\right)
$$

achieves its only minimum at $r=1$ if $\hat{\eta}$ is small enough. This is a problem on a real line and the desired result can be obtained by examining the derivative of $g$ in separate cases $r \in(0,1)$ and $r \in[1, R]$. 
For more detail look at the proofs of Lemma 4.1 and Lemma 4.2 in [DPMM19]. The computation can be repeated almost verbatim, the only difference being scaling of capacity.

4.2. A penalized minimum problem. The sequence in Theorem 3.2 is obtained by solving the following minimum problem (see also [AFM14] where a similar penalized problem is introduced to deal with a nonlocal isoperimetric problem).

$$
\min \left\{\mathscr{C}_{\hat{\eta}, j}(\Omega): \Omega \subset B_{R}\right\}
$$

where

$\mathscr{C}_{\hat{\eta}, j}(\Omega)=\mathscr{C}_{\hat{\eta}}(\Omega)+\sqrt{\varepsilon_{j}^{2}+\sigma^{2}\left(\alpha(\Omega)-\varepsilon_{j}\right)^{2}}=\operatorname{cap}_{p}(\Omega)+f_{\hat{\eta}}(|\Omega|)+\sqrt{\varepsilon_{j}^{2}+\sigma^{2}\left(\alpha(\Omega)-\varepsilon_{j}\right)^{2}}$.

Lemma 4.2. There exists $\sigma_{0}=\sigma_{0}(N, R)>0$ such that for every $\sigma<\sigma_{0}$ the minimum in (4.2) is attained by a p-quasi-open ${ }^{3}$ set $\Omega_{j}$. Moreover, perimeters of $\Omega_{j}$ are bounded independently on $j$.

Proof. The proof is almost verbatim repetition of the proof of [DPMM19, Lemma 4.3].

Step 1: finding minimizing sequence with bounded perimters. We consider $\left\{V_{k}\right\}_{k \in \mathbb{N}}$ - a minimizing sequence for $\mathscr{C}_{\hat{\eta}, j}$, satisfying

$$
\mathscr{C}_{\hat{\eta}, j}\left(V_{k}\right) \leq \inf \mathscr{C}_{\hat{\eta}, j}+\frac{1}{k}
$$

We denote by $v_{k}$ the capacitary potentials of $V_{k}$, so $V_{k}=\left\{x \in B_{R}: v_{k}=1\right\}$. We take as a variation the slightly enlarged set $\tilde{V}_{k}$ :

$$
\tilde{V}_{k}=\left\{x \in B_{R}: v_{k}>1-t_{k}\right\},
$$

where $t_{k}=\frac{1}{\sqrt{k}}$.

Note that the function $\tilde{v}_{k}=\frac{\min \left(v_{k}, 1-t_{k}\right)}{1-t_{k}}$ is in $D^{1, p}\left(\mathbb{R}^{N}\right)$ and $v_{k}=1$ on $\tilde{V}_{k}$, so we can bound the p-capacity of $\tilde{V}_{k}$ by $\int\left|\nabla \tilde{v}_{k}\right|^{p} d x$. Since $V_{k}$ is almost minimizing, we write

$$
\begin{aligned}
& \int_{\left\{v_{k}<1\right\}}\left|\nabla v_{k}\right|^{p} d x+f_{\hat{\eta}}\left(\left|\left\{v_{k}=1\right\}\right|\right)+\sqrt{\varepsilon_{j}^{2}+\sigma^{2}\left(\alpha\left(\left\{v_{k}=1\right\}\right)-\epsilon_{j}\right)^{2}} \\
& \leq \int_{\left\{v_{k}<1-t_{k}\right\}}\left|\nabla\left(\frac{v_{k}}{1-t_{k}}\right)\right|^{p} d x+f_{\hat{\eta}}\left(\left|\left\{v_{k} \geq 1-t_{k}\right\}\right|\right)+\sqrt{\varepsilon_{j}^{2}+\sigma^{2}\left(\alpha\left(\left\{v_{k} \geq 1-t_{k}\right\}\right)-\varepsilon_{j}\right)^{2}}+\frac{1}{k} .
\end{aligned}
$$

\footnotetext{
${ }^{3}$ Recall that a set is said to be $p$-quasi-open if it is the zero level set of a $W^{1, p}$ function.
} 
We use (4.1) and the fact that the function $t \mapsto \sqrt{\varepsilon_{j}^{2}+\sigma^{2}\left(t-\varepsilon_{j}\right)^{2}}$ is 1 Lipschitz to get

$$
\begin{aligned}
& \int_{\left\{1-t_{k}<v_{k}<1\right\}}\left|\nabla v_{k}\right|^{p} d x+\hat{\eta}\left|\left\{1-t_{k}<v_{k}<1\right\}\right| \\
& \leq \sigma\left(\left.\left|\alpha\left(\left\{v_{k} \geq 1-t_{k}\right\}-\alpha\left(\left\{v_{k}=1\right\}\right) \mid\right)+\frac{1}{k}+\int_{\left\{v_{k}<1-t_{k}\right\}}\left(\left(\frac{1}{1-t_{k}}\right)^{p}-1\right)\right| \nabla v_{k}\right|^{p} d x\right. \\
& \leq C(R) \sigma\left|\left\{1-t_{k}<v_{k} \leq 1\right\}\right|+\frac{1}{k}+\left(\left(\frac{1}{1-t_{k}}\right)^{p}-1\right) \operatorname{cap}_{p}\left(V_{k}\right) \\
& \leq C(R) \sigma\left|\left\{1-t_{k}<v_{k} \leq 1\right\}\right|+\frac{1}{k}+c(N, R) t_{k},
\end{aligned}
$$

where in the second inequality we used Lemma 2.3, (ii). Taking $\sigma<\frac{\hat{\eta}}{2 C(R)}$, we obtain

$$
\int_{\left\{1-t_{k}<v_{k}<1\right\}}\left|\nabla v_{k}\right|^{p} d x+\frac{\hat{\eta}}{2}\left(\left|\left\{1-t_{k}<v_{k}<1\right\}\right|\right) \leq \frac{1}{k}+c(N, R) t_{k} .
$$

We estimate the left-hand side from below, using the arithmetic-geometric mean inequality, the Cauchy-Schwarz inequality, and the co-area formula.

$$
\begin{aligned}
& \int_{\left\{1-t_{k}<v_{k}<1\right\}}\left|\nabla v_{k}\right|^{p} d x+\frac{\hat{\eta}}{2}\left(\left|\left\{1-t_{k}<v_{k}<1\right\}\right|\right) \\
& \geq p\left(\int_{1-t_{k}<v_{k}<1}\left|\nabla v_{k}\right|^{p} d x\right)^{\frac{1}{p}}\left((p-1) \frac{\hat{\eta}}{2}\left(\left|\left\{1-t_{k}<v_{k}<1\right\}\right|\right)\right)^{\frac{1}{p^{\prime}}} \\
& \geq c \hat{\eta}^{\frac{1}{p^{\prime}}} \int_{1-t_{k}<v_{k}<1}\left|\nabla v_{k}\right| d x=c \hat{\eta}^{\frac{1}{p^{\prime}}} \int_{1-t_{k}}^{1} P\left(\left\{v_{k}>s\right\}\right) d s .
\end{aligned}
$$

where $P(E)$ denotes the De Giorgi perimeter of a set $E$. Hence, there exists a level $1-t_{k}<s_{k}<1$ such that for $\hat{V}_{k}=\left\{v_{k}>s_{k}\right\}$

$$
P\left(\hat{V}_{k}\right) \leq \frac{1}{t_{k}} \int_{1-t_{k}}^{1} P\left(\left\{v_{k}>s\right\}\right) d s \leq \frac{1}{t_{k} c \hat{\eta}^{\frac{1}{p^{\prime}} k}}+c(N, R)=\frac{1}{c \hat{\eta}^{\frac{1}{p^{\prime}}} \sqrt{k}}+c(N, R) .
$$

where in the last equality we have used that $t_{k}=\frac{1}{\sqrt{k}}$. These $\hat{V}_{k}$ will give us the desired "good" minimizing sequence, indeed

$$
\begin{aligned}
\mathscr{C}_{\hat{\eta}, j}\left(\hat{V}_{k}\right) \leq \mathscr{C}_{\hat{\eta}, j}\left(V_{k}\right)+f_{\hat{\eta}}\left(\left|\left\{v_{k}>s_{k}\right\}\right|\right) & -f_{\hat{\eta}}\left(\left|\left\{v_{k}=1\right\}\right|\right) \\
& +C \sigma\left|\left\{1-s_{k}<v_{k}<1\right\}\right| \leq \mathscr{C}_{\hat{\eta}, j}\left(V_{k}\right),
\end{aligned}
$$

where in the first inequality we have used that $\hat{V}_{k} \subset V_{k}$ and in the second that, thanks to our choice of $\sigma$,

$f_{\hat{\eta}}\left(\left|\left\{v_{k}>s_{k}\right\}\right|\right)-f_{\hat{\eta}}\left(\left|\left\{v_{k}=1\right\}\right|\right)+C \sigma\left|\left\{1-s_{k}<v_{k}<1\right\} \leq(C \sigma-\hat{\eta})\right|\left\{1-s_{k}<v_{k}<1\right\} \leq 0$.

Step 2: Existence of a minimizer. Since $\left\{\hat{V}_{k}\right\}_{k}$ is a sequence with equibounded perimeter,s there exists a Borel set $\hat{V}_{\infty}$ such that up to a (not relabelled) subsequence

$$
1_{\hat{V}_{k}} \rightarrow 1_{\hat{V}_{\infty}} \text { in } L_{1}\left(B_{R}\right) \text { and a.e. in } B_{R}, \quad P\left(\hat{V}_{\infty}\right) \leq C(N, R) .
$$


We want to show that $\hat{V}_{\infty}$ is a minimizer for $\mathcal{C}_{\eta, j}$. We set $\hat{v}_{k}=\frac{\min \left(v_{k}, s_{k}\right)}{s_{k}}$ and we note that they are the capacitary potentials of $\hat{V}_{k}$. Moreover the sequence $\left\{\hat{v}_{k}\right\}_{k}$ is bounded in $D^{1, p}\left(\mathbb{R}^{N}\right)$. Thus, there exists a function $\hat{v} \in D^{1, p}\left(\mathbb{R}^{N}\right)$ such that up to a (not relabelled) subsequence

$$
\hat{v}_{k} \rightarrow \hat{v} \text { strongly in } L^{p}\left(B_{R}\right) \text { and a.e. in } B_{R} .
$$

Let us define $\hat{V}=\{x: \hat{v}=1\}$, we want to show that $\hat{V}$ is a minimizer. First, note that

$$
1_{\hat{V}}(x) \geq \limsup 1_{\hat{V}_{k}}(x)=1_{\hat{V}_{\infty}}(x) \quad \text { for a.e. } x \in B_{R},
$$

hence $\left|\hat{V}_{\infty} \backslash \hat{V}\right|=0$. Moreover, by the lower semicontinuity of Dirichlet integral, the monotonicity of $f_{\hat{\eta}}$ and the continuity of $\alpha$ with respect to the $L^{1}$ convergence, we have

$$
\begin{aligned}
\inf \mathscr{C}_{\hat{\eta}, j} & =\lim _{k} \int\left|\nabla \hat{v}_{k}\right|^{p}+f_{\hat{\eta}}\left(\left|\hat{V}_{k}\right|\right)+\sqrt{\varepsilon_{j}^{2}+\sigma^{2}\left(\alpha\left(\hat{V}_{k}\right)-\varepsilon_{j}\right)^{2}} \\
& \geq \operatorname{cap}_{p}(\hat{V})+f_{\hat{\eta}}\left(\left|\hat{V}_{\infty}\right|\right)+\sqrt{\varepsilon_{j}^{2}+\sigma^{2}\left(\alpha\left(\hat{V}_{\infty}\right)-\varepsilon_{j}\right)^{2}} \geq \operatorname{cap}_{p}(\hat{V})+f_{\hat{\eta}}(|\hat{V}|) .
\end{aligned}
$$

Hence

$$
\begin{aligned}
\operatorname{cap}_{p}(\hat{V}) & +f_{\hat{\eta}}\left(\left|\hat{V}_{\infty}\right|\right)+\sqrt{\varepsilon_{j}^{2}+\sigma^{2}\left(\alpha\left(\hat{V}_{\infty}\right)-\varepsilon_{j}\right)^{2}} \leq \inf \mathscr{C}_{\hat{\eta}, j}^{R}(\Omega) \\
& \leq \operatorname{cap}_{p}(\hat{V})+f_{\hat{\eta}}(|\hat{V}|)+\sqrt{\varepsilon_{j}^{2}+\sigma^{2}\left(\alpha(\hat{V})-\varepsilon_{j}\right)^{2}}
\end{aligned}
$$

Using Lemma 2.3 (ii) we get

$$
f_{\hat{\eta}}\left(\left|\hat{V}_{\infty}\right|\right)-f_{\hat{\eta}}(|\hat{V}|) \leq C \sigma\left|\hat{V} \Delta \hat{V}_{\infty}\right|=C \sigma\left|\hat{V} \backslash \hat{V}_{\infty}\right| .
$$

Since $|\hat{V}| \geq\left|\hat{V}_{\infty}\right|,(4.1)$ and our choice of $\sigma$ yield

$$
\hat{\eta}\left|\hat{V} \backslash \hat{V}_{\infty}\right| \leq f_{\hat{\eta}}\left(\left|\hat{V}_{\infty}\right|\right)-f_{\hat{\eta}}(|\hat{V}|) \leq C \sigma\left|\hat{V} \backslash \hat{V}_{\infty}\right| \leq \frac{\hat{\eta}}{2}\left|\hat{V} \backslash \hat{V}_{\infty}\right|
$$

from which we conclude that $\left|\hat{V} \Delta \hat{V}_{\infty}\right|=0$ and thus, by (4.3) that $\hat{V}$ is the desired minimizer.

\subsection{First properties of the minimizers.}

Lemma 4.3. Let $\left\{\Omega_{j}\right\}$ be a sequence of minimizers for (4.2). Then the following properties hold:

(i) $\left|\alpha\left(\Omega_{j}\right)-\varepsilon_{j}\right| \leq 3 \sigma \varepsilon_{j}$;

(ii) ||$\Omega_{j}|-| B_{1}|| \leq C \sigma^{4} \varepsilon_{j}$;

(iii) up to translations $\Omega_{j} \rightarrow B_{1}$ in $L^{1}$,

(iv) $0 \leq \mathscr{C}_{\hat{\eta}}\left(\Omega_{j}\right)-\mathscr{C}_{\hat{\eta}}\left(B_{1}\right) \leq \sigma^{4} \varepsilon_{j}$.

Proof. The lemma follows easily from Lemma 4.1. To prove (iii) we need to recall that the sets $\Omega_{j}$ have bounded perimeter.

For more details see the proof of [DPMM19, Lemma 4.4]. 


\section{Proof of Theorem 3.2: Regularity}

In this section, we show that the sequence of minimizers of (4.2) converges smoothly to the unit ball. This will be done by relying on the regularity theory for free boundary problems established in [DP05].

5.1. Linear growth away from the free boundary. Let $u_{j}$ be the capacitary potential for $\Omega_{j}$, a minimizer of (4.2). Let us also introduce $v_{j}:=1-u_{j}$, so that $\Omega_{j}=\left\{v_{j}=0\right\}$. Following [DP05] we are going to show that

$$
v_{j}(x) \sim \operatorname{dist}\left(x, \Omega_{j}\right) .
$$

where the implicit constant depends only on $R$. The above estimate is obtained by suitable comparison estimates. We will need to have some compactness properties, so we first prove Hölder continuity, also with the constant depending only on $R$.

5.1.1. Hölder continuity. The proof is based on establishing a decay estimate for the integral oscillation of $u_{j}$ and it is almost identical to the case of 2-capacity (see [DPMM19, Lemma 5.8]).

We are going to use the following growth result for $p$-harmonic functions. The proof can be found, for example, in [G, Theorem 7.7].

Lemma 5.1. Suppose $w \in W^{1,2}(\Omega)$ is p-harmonic, $x_{0} \in \Omega$. Then there exists a constant $c=c(N, p), 0<\beta \leq 1$ such that for any balls $B_{r_{1}}\left(x_{0}\right) \subset B_{r_{2}}\left(x_{0}\right) \Subset \Omega$

$$
f_{B_{r_{1}}\left(x_{0}\right)}|\nabla w|^{p} \leq c\left(\frac{r_{1}}{r_{2}}\right)^{p \beta-p} f_{B_{r_{2}}\left(x_{0}\right)}|\nabla w|^{p} .
$$

Remark 5.2. In $[G]$ the result is proven for the functions in De Giorgi class. One can prove that in the case of $p$-harmonic functions the inequality holds for $\beta=1$, but we are not going to need that.

To prove Hölder continuity of $u_{j}$ we will use several times the following comparison estimates.

Lemma 5.3. Let $u_{j}$ be the capacitary potential of a minimizer for (4.2). Let $A \subset B_{R}$ be an open set with Lipschitz boundary and let $w \in W^{1, p}\left(\mathbb{R}^{n}\right)$ coincide with $u_{j}$ on the boundary of $A$ in the sense of traces.

Then

$$
\int_{A}\left|\nabla u_{j}\right|^{p} d x-\int_{A}|\nabla w|^{p} d x \leq\left(\frac{1}{\hat{\eta}}+C \sigma\right)|A \cap(\{u=1\} \Delta\{w=1\})| .
$$

Moreover, if $u_{j} \leq w \leq 1$ in $A$, then

$$
\int_{A}\left|\nabla u_{j}\right|^{p} d x+\frac{\hat{\eta}}{2}|A \cap(\{u=1\} \Delta\{w=1\})| \leq \int_{A}|\nabla w|^{p} d x
$$

provided $\sigma \leq \sigma(N, R)$.

Proof. The proof is the same as the proof of [DPMM19, Lemma 5.5], modulo changing exponents from 2 to $p$. The idea is to consider $\tilde{u}$ defined as

$$
\begin{cases}\tilde{u}=w & \text { in } A \\ \tilde{u}=u & \text { else }\end{cases}
$$


and take $\tilde{\Omega}=\{\tilde{u}=1\}$ as a comparison domain.

Remark 5.4. Note that if $w$ is $p$-harmonic in $A$, then by Lemma A.2

- if $p \geq 2$, we have

$$
\int_{A}|\nabla u|^{p} d x-\int_{A}|\nabla w|^{p} d x \geq c \int_{A}|\nabla(u-w)|^{p} d x
$$

- if $1<p<2$, then

$$
\int_{A}|\nabla u|^{p} d x-\int_{A}|\nabla w|^{p} d x \geq c \int_{A}|\nabla(u-w)|^{2}\left(|\nabla w|^{2}+|\nabla(u-w)|^{2}\right)^{\frac{p-2}{2}} d x .
$$

Hence the first inequality from the lemma becomes

- for $p \geq 2$

$$
\int_{A}|\nabla(u-w)|^{p} d x \leq C(p)\left(\frac{1}{\hat{\eta}}+C \sigma\right)|A \cap(\{u=1\} \Delta\{w=1\})| ;
$$

- for $1<p<2$

$$
\begin{aligned}
& \int_{A}|\nabla(u-w)|^{2}\left(|\nabla w|^{2}+|\nabla(u-w)|^{2}\right)^{\frac{p-2}{2}} d x \\
& \quad \leq C(p)\left(\frac{1}{\hat{\eta}}+C \sigma\right)|A \cap(\{u=1\} \Delta\{w=1\})| .
\end{aligned}
$$

Let us also recall the following technical result.

Lemma 5.5 (Lemma 5.13 in $[\mathrm{GM}]$ ). Let $\phi: \mathbb{R}^{+} \rightarrow \mathbb{R}^{+}$be a non-decreasing function satisfying

$$
\phi(\rho) \leq A\left[\left(\frac{\rho}{R}\right)^{\alpha}+\varepsilon\right] \phi(R)+B R^{\beta},
$$

for some $A, \alpha, \beta>0$, with $\alpha>\beta$ and for all $0<\rho \leq R \leq R_{0}$, where $R_{0}>0$ is given. Then there exist constants $\varepsilon_{0}=\varepsilon_{0}(A, \alpha, \beta)$ and $c=c(A, \alpha, \beta)$ such that if $\varepsilon \leq \varepsilon_{0}$, we have

$$
\phi(\rho) \leq c\left[\frac{\phi(R)}{R^{\beta}}+B\right] \rho^{\beta}
$$

for all $0 \leq \rho \leq R \leq R_{0}$.

We are now ready to prove Hölder continuity of $u_{j}$.

Lemma 5.6. There exists $\alpha \in(0,1 / 2)$ such that the capacitary potential of every minimizer of (4.2) satisfies $u_{j} \in C^{0, \alpha}\left(\overline{B_{R}}\right)$. Moreover, the Hölder norm is bounded by a constant independent on $j$.

Proof. The proof is similar to the proof of [DPMM19, Lemma 5.8]. As usual, we drop the subscript $j$. By Morrey Theorem (see, for example, [GM, Theorem 5.7]) it is enough to show that

$$
\phi(r):=\int_{B_{r}\left(x_{0}\right)}|\nabla u|^{p} \leq C r^{N+2 \alpha-p}
$$

for all $r$ small enough (say less that $1 / 2$ ). 
Let $x_{0} \in B_{R}$. Let $w$ be the p-harmonic extension of $u$ in $B_{r^{\prime}}\left(x_{0}\right)$. By Lemma 5.1 we know that

$$
\int_{B_{r}\left(x_{0}\right)}|\nabla w|^{p} \leq C\left(\frac{r}{r^{\prime}}\right)^{N+p \beta-p} \int_{B_{r^{\prime}}\left(x_{0}\right)}|\nabla w|^{p}
$$

Let $g:=u-w$. Then

$$
\begin{aligned}
& \int_{B_{r}\left(x_{0}\right)}|\nabla u|^{p} d x \leq C \int_{B_{r}\left(x_{0}\right)}|\nabla w|^{p} d x+C \int_{B_{r}\left(x_{0}\right)}|\nabla g|^{p} \\
& \leq C\left(\frac{r}{r^{\prime}}\right)^{N+p \beta-p} \int_{B_{r^{\prime}}\left(x_{0}\right)}|\nabla w|^{p} d x+C \int_{B_{r^{\prime}}\left(x_{0}\right)}|\nabla g|^{p} .
\end{aligned}
$$

We want to show the following bound:

$$
\int_{B_{r^{\prime}}\left(x_{0}\right)}|\nabla g|^{p} \leq C_{\varepsilon}\left(r^{\prime}\right)^{N}+C \varepsilon \int_{B_{r^{\prime}}\left(x_{0}\right)}|\nabla w|^{p}
$$

for $\varepsilon<\varepsilon_{0}=\varepsilon_{0}(N, p)$. By (5.1) it is immediate for $p \geq 2$ (even without the second summand on the right hand side). For $1<p<2$ we use Young inequality (in the form $a b \leq C_{\varepsilon} a^{q}+\varepsilon b^{q^{\prime}}$ with $\left.q=2 / p\right)$ to get

$$
\begin{array}{r}
\int_{B_{r^{\prime}}\left(x_{0}\right)}|\nabla g|^{p}=\int_{B_{r^{\prime}}\left(x_{0}\right)}|\nabla g|^{p}\left(|\nabla w|^{2}+|\nabla g|^{2}\right)^{p(p-2) / 4}\left(|\nabla w|^{2}+|\nabla g|^{2}\right)^{-p(p-2) / 4} \\
\leq C_{\varepsilon} \int_{B_{r^{\prime}}\left(x_{0}\right)}|\nabla g|^{2}\left(|\nabla w|^{2}+|\nabla g|^{2}\right)^{(p-2) / 2}+\varepsilon \int_{B_{r^{\prime}}\left(x_{0}\right)}\left(|\nabla w|^{2}+|\nabla g|^{2}\right)^{p / 2} \\
\leq C_{\varepsilon}\left(r^{\prime}\right)^{N}+C \varepsilon \int_{B_{r^{\prime}}\left(x_{0}\right)}\left(|\nabla w|^{p}+|\nabla g|^{p}\right),
\end{array}
$$

yielding (5.3) for $\varepsilon$ small enough. Note that in the last inequality we used (5.2). So we have

$$
\begin{gathered}
\int_{B_{r}\left(x_{0}\right)}|\nabla u|^{p} d x \leq C\left(\left(\frac{r}{r^{\prime}}\right)^{N+p \beta-p}+\varepsilon\right) \int_{B_{r^{\prime}}\left(x_{0}\right)}|\nabla w|^{p} d x+C_{\varepsilon}\left(r^{\prime}\right)^{N} \\
\leq C\left(\left(\frac{r}{r^{\prime}}\right)^{N+p \beta-p}+\varepsilon\right) \int_{B_{r^{\prime}\left(x_{0}\right)}}|\nabla u|^{p} d x+C_{\varepsilon}\left(r^{\prime}\right)^{N} \\
+C\left(\left(\frac{r}{r^{\prime}}\right)^{N+p \beta-p}+\varepsilon\right) \int_{B_{r^{\prime}}\left(x_{0}\right)}|\nabla g|^{p} d x \\
\leq C\left(\left(\frac{r}{r^{\prime}}\right)^{N+p \beta-p}+\varepsilon\right) \int_{B_{r^{\prime}}\left(x_{0}\right)}|\nabla u|^{p} d x+C_{\varepsilon}\left(r^{\prime}\right)^{N},
\end{gathered}
$$

which gives us

$$
\phi(r) \leq c\left(\left(\frac{r}{r^{\prime}}\right)^{N+p \beta-p}+\varepsilon\right) \phi\left(r^{\prime}\right)+C_{\varepsilon}\left(r^{\prime}\right)^{N} .
$$

Using Lemma 5.5 we obtain

$$
\phi(r) \leq c\left(\left(\frac{r}{r^{\prime}}\right)^{N+p \beta-p} \phi\left(r^{\prime}\right)+C r^{N}\right)
$$

for any $r<r^{\prime}<1$. In particular,

$$
\phi(r) \leq c\left(\|u\|_{L^{p}\left(\mathbb{R}^{N}\right)}^{p}+C\right) r^{N+p \beta-p} .
$$


5.1.2. Lipschitz continuity and density estimates on the boundary. The following lemma is an analogue of [DP05, Lemma 3.2] and it will give us uniform Lipschitz continuity.

Lemma 5.7. There exists $M=M(N, R)$ such that if $u_{j}$ is the capacitary potential of a minimizer for (4.2) and $v_{j}=1-u_{j}$ satisfies $v_{j}\left(x_{0}\right)=0$, then

$$
\sup _{B_{r / 4}\left(x_{0}\right)} v_{j} \leq M r
$$

Proof. Step 1. We argue by contradiction and get a sequence $v_{j_{k}}, B_{r_{k}}\left(y_{k}\right) \subset B_{R}$ such that $v_{j_{k}}\left(y_{k}\right)=0, \sup _{B_{r_{k} / 4}\left(y_{k}\right)} v_{j_{k}} \geq k r_{k}$. We now consider blow-ups around $y_{k}$, that is, we define

$$
\tilde{v}_{k}(x):=\frac{v_{j_{k}}\left(y_{k}+r_{k} x\right)}{r_{k}}
$$

Note that $\tilde{v}_{k}$ minimizes

$$
\int_{\mathbb{R}^{N}}|\nabla v|^{p} d x+r_{k}^{-N} f_{\hat{\eta}}\left(r_{k}^{N}\{v=0\}\right)+r_{k}^{-N} \sqrt{\varepsilon_{j_{k}}^{2}+\sigma^{2}\left(\alpha\left(\Phi_{k}(\{v=0\})\right)-\varepsilon_{j_{k}}\right)^{2}}
$$

among functions such that $\Phi_{k}(\{v=0\}) \subset B_{R}$, where $\Phi_{k}(x)=y_{k}+r_{k} x$. Additionally, we have

$$
\tilde{v}_{k}(0)=0, \sup _{B_{1 / 4}} \tilde{v}_{k} \geq k
$$

We define a function

$$
d_{k}(x):=\operatorname{dist}\left(x,\left\{\tilde{v}_{k}=0\right\}\right)
$$

and a set

$$
V_{k}:=\left\{x \in B: d_{k}(x) \leq \frac{1-|x|}{3}\right\} .
$$

The following properties hold for $V_{k}$ :

- $B_{1 / 4} \subset V_{k}$. This is due to the fact that $\tilde{v}_{k}(0)=0$ and thus $d_{k}(x) \leq|x|$.

- $m_{k}:=\sup _{x \in V_{k}}(1-|x|) \tilde{v}_{k}(x) \geq \frac{3 k}{4}$. This follows from the previous property and the fact that $\sup _{B_{1 / 4}} \tilde{v}_{k} \geq k$.

Since $\tilde{v}_{k}$ is continuous and $(1-|x|) \tilde{v}_{k}(x)=0$ on $\partial B, m_{k}$ is obtained at some point $x_{k} \in V_{k}$. We notice that the following holds for $x_{k}$ :

$$
\tilde{v}_{k}\left(x_{k}\right)=\frac{m_{k}}{1-\left|x_{k}\right|} \geq m_{k} \geq \frac{3 k}{4} ; \quad \delta_{k}:=d_{k}\left(x_{k}\right) \leq \frac{1-\left|x_{k}\right|}{3} .
$$

We now take projections of $x_{k}$ onto $\left\{\tilde{v}_{k}=0\right\}$, that is, we consider a sequence $z_{k}$ such that $z_{k} \in\left\{\tilde{v}_{k}=0\right\} \cap B,\left|z_{k}-x_{k}=\delta_{k}\right|$. Note that $B_{2 \delta_{k}}\left(z_{k}\right) \subset B$. Moreover, $B_{\delta_{k} / 2}\left(z_{k}\right) \subset V_{k}$ since for any $x \in B_{\delta_{k} / 2}\left(z_{k}\right)$ we have

$$
1-|x| \geq 1-\left|x_{k}\right|-\left|x_{k}-x\right| \geq 1-\left|x_{k}\right|-\frac{3}{2} \delta_{k} \geq \frac{1-\left|x_{k}\right|}{2} .
$$


Now let us show that $\sup _{B_{\delta_{k}} / 4} \tilde{v}_{k} \sim \tilde{v}_{k}\left(x_{k}\right)$. Indeed, for the upper bound it is enough to notice that

$$
\sup _{B_{\delta_{k} / 2}\left(z_{k}\right)} \tilde{v}_{k} \leq \tilde{v}_{k}\left(x_{k}\right)\left(1-\left|x_{k}\right|\right) \sup _{B_{\delta_{k} / 2}\left(z_{k}\right)} \frac{1}{1-|x|} \leq 2 \tilde{v}_{k}\left(x_{k}\right) .
$$

On the other hand, since $B_{\delta_{k}}\left(x_{k}\right) \subset\left\{\tilde{v}_{k}>0\right\}, \tilde{v}_{k}$ is $p$-harmonic in $B_{\delta_{k}}\left(x_{k}\right)$ and thus, by Harnack inequality (see, for example, [L06, Theorem 2.20]), we get

$$
\sup _{B_{\delta_{k} / 4}\left(z_{k}\right)} \tilde{v}_{k} \geq \inf _{B_{4 \delta_{k} / 5}\left(x_{k}\right)} \tilde{v}_{k} \geq c_{0} \sup _{B_{4 \delta_{k} / 5}\left(x_{k}\right)} \tilde{v}_{k}-C \geq \frac{c_{0}}{2} \tilde{v}_{k}\left(x_{k}\right),
$$

where the last inequality holds for $k$ big enough.

Step 2. We now consider blow-ups around $z_{k}$. We define

$$
\hat{v}_{k}(x):=\frac{\tilde{v}_{k}\left(z_{k}+\frac{\delta_{k}}{2} x\right)}{\tilde{v}_{k}\left(x_{k}\right)} \text {. }
$$

We note that

$$
\sup _{B} \hat{v}_{k} \leq 2, \quad \sup _{B_{1 / 2}} \hat{v}_{k} \geq c_{0} / 2, \quad \hat{v}_{k}(0)=0
$$

and $\hat{v}_{k}$ is a minimizer of

$$
\begin{array}{r}
\int_{\mathbb{R}^{n}}|\nabla v|^{p} d x+\left(\delta_{k} / 2\right)^{p-n} \tilde{v}_{k}\left(x_{k}\right)^{-p} r_{k}^{-n} f_{\hat{\eta}}\left(\left(\delta_{k} / 2\right)^{n} r_{k}^{n}\{v=0\}\right)+ \\
\quad\left(\delta_{k} / 2\right)^{p-n} \tilde{v}_{k}\left(x_{k}\right)^{-p} r_{k}^{-n} \sqrt{\varepsilon_{j_{k}}^{2}+\sigma^{2}\left(\alpha\left(\Psi_{k}(\{v=0\})\right)-\varepsilon_{j_{k}}\right)^{2}}
\end{array}
$$

among functions such that $\Psi_{k}(\{v=0\}) \subset B_{R}$, where $\Psi_{k}(x)=y_{k}+r_{k} z_{k}+\frac{r_{k} \delta_{k} x}{2}$.

We introduce $w_{k}$ - a $p$-harmonic continuation of $\hat{v}_{k}$ in $B_{3 / 4}$ :

$$
\left\{\begin{array}{l}
\operatorname{div}\left(\nabla\left|w_{k}\right|^{p-2} \nabla w_{k}\right)=0 \text { in } B_{3 / 4}, \\
w_{k}=\hat{v}_{k} \text { in } B_{3 / 4}^{c}
\end{array}\right.
$$

By maximum principle (see, for example, [L06, Corollary 2.21]) $w_{k}>0$ in $B_{3 / 4}$ and thus

$$
\left\{\hat{v}_{k}=0\right\} \Delta\left\{w_{k}=0\right\}=\left\{\hat{v}_{k}=0\right\} \cap B_{3 / 4} .
$$

So now, remembering that $\hat{v}_{k}$ is a minimizer for (5.4) and using $w_{k}$ as a comparison function we obtain

$$
\int_{B_{3 / 4}}\left|\nabla \hat{v}_{k}\right|^{p} d x \leq \int_{B_{3 / 4}}\left|\nabla w_{k}\right|^{p} d x+\frac{C}{k^{p}} .
$$

From this we can infer the convergence of $v_{k}-w_{k}$ to zero. In order to do that, we define

$$
v_{k}^{s}=s \hat{v}_{k}+(1-s) w_{k} .
$$

Now, we write

$$
\begin{aligned}
& \frac{C}{k^{p}} \geq \int_{B_{3 / 4}}\left|\nabla \hat{v}_{k}\right|^{p} d x-\int_{B_{3 / 4}}\left|\nabla w_{k}\right|^{p} d x=p \int_{0}^{1} \frac{1}{s} d s \int_{B_{3 / 4}}\left|\nabla v_{k}^{s}\right|^{p-2} \nabla v_{k}^{s} \cdot \nabla\left(v_{k}^{s}-w_{k}\right) d x \\
&=p \int_{0}^{1} \frac{1}{s} d s \int_{B_{3 / 4}}\left(\left|\nabla v_{k}^{s}\right|^{p-2} \nabla v_{k}^{s}-\left|\nabla w_{k}\right|^{p-2} \nabla w_{k}\right) \cdot \nabla\left(v_{k}^{s}-w_{k}\right) d x .
\end{aligned}
$$


We want to show that the convergence of $v_{k}-w_{k}$ is strong. We use Lemma A.1 for that. We need to consider two cases. For $p \geq 2$ by the inequality (A.1) we get

$$
\int_{B_{3 / 4}}\left|\nabla \hat{v}_{k}-\nabla w_{k}\right|^{p} d x \leq \frac{C}{k^{p}}
$$

yielding the strong convergence of $\hat{v}_{k}-w_{k}$ to zero in $W^{1, p}\left(B_{3 / 4}\right)$ as $k \rightarrow \infty$. To deal with the case $1<p<2$, we observe that $\hat{v}_{k}$ is bounded in $D^{1, p}\left(B_{3 / 4}\right)$. We infer that $w_{k}$ is bounded in $D^{1, p}$ too and hence, by the inequality (A.2) we also have the strong convergence of $\hat{v}_{k}-w_{k}$ to zero in $W^{1, p}\left(B_{3 / 4}\right)$ as $k \rightarrow \infty$.

We recall now that $\hat{v}_{k}$ is equibounded in $C^{0, \alpha}\left(B_{3 / 4}\right)$ and hence, up to a nonrelabelled subsequence we have that $\hat{v}_{k}$ converges to some continuous function $v_{\infty}$ locally uniformly and weakly in $W^{1, p}$. This means that also $w_{k}$ converges to $v_{\infty}$ weakly in $W^{1, p}$. Elliptic regularity for $w_{k}$ tell us that $w_{k}$ is locally bounded in $C^{1, \beta}\left(B_{3 / 4}\right)$ and so up to a subsequence $w_{k}$ converges to $v_{\infty}$ strongly in $W^{1, p}$. But then $v_{\infty}$ is $p$-harmonic with $v_{\infty}(0)=0, \sup _{B_{1 / 2}} v_{\infty} \geq c_{0} / 2$. This contradicts Harnack inequality.

The following lemma is an analogue of [DP05, Lemma 4.2] and the proof is almost identical.

Lemma 5.8 (non-degeneracy). For $\kappa<1, \gamma>p-1$ there exists a constant $c_{n d}=c_{n d}(N, \kappa, \gamma, R)$ such that if $u_{j}$ is a minimizer for (4.2) and $v_{j}=1-u_{j}$ satisfies

$$
\left(f_{\partial B_{r}\left(x_{0}\right)} v_{j}^{\gamma}\right)^{\frac{1}{\gamma}} \leq c r
$$

then $v_{j}=0$ in $B_{\kappa r}\left(x_{0}\right)$.

Proof. We will omit the subscript $j$ for convenience and write $v$ instead of $v_{j}$. None of the bounds will depend on $j$.

First, we want to show that if (5.5) holds for $\partial B_{r}\left(x_{0}\right)$ and $c$ is small enough (depending only on $N, \kappa, \gamma$, and $R$ ), then the inequality (5.5) yields $B_{\kappa r} \subset B_{R}$. The idea is that $v$ is sufficiently big outside of $B_{R}$. Indeed, by maximum principle

$$
v(x) \geq 1-u_{B_{R}}(x)=1-\frac{R^{\frac{n-p}{p-1}}}{|x|^{\frac{n-p}{p-1}}} .
$$

If $B_{\kappa r}\left(x_{0}\right) \backslash B_{R} \neq \emptyset$, then $\left|B_{r}\left(x_{0}\right) \backslash B_{R+\frac{1-\kappa}{2} r}\right| \geq c(\kappa)\left|B_{r}\right|$ and, using (5.6), we get

$$
\left(f_{\partial B_{r}\left(x_{0}\right)} v_{j}^{\gamma}\right)^{\frac{1}{\gamma}} \geq c(\kappa)\left(1-\frac{R^{\frac{n-p}{p-1}}}{\left|R+\frac{1-\kappa}{2} r\right|^{\frac{n-p}{p-1}}}\right) \geq c(\kappa, N, R) r
$$

contradicting (5.5) for $c_{n d}$ small enough.

Now we define

$$
\varepsilon:=\frac{1}{\sqrt{\kappa} r} \sup _{B_{\sqrt{\kappa} r}} v \leq C \frac{1}{r}\left(f_{\partial B_{r}\left(x_{0}\right)} v^{\gamma}\right)^{\frac{1}{\gamma}} \leq C c_{n d},
$$


where we used Harnack inequality for $p$-subharmonic functions (see [T67, Theorem 1.3]). We set $\varphi(x)=\varphi(|x|)$ to be the solution of

$$
\begin{cases}\Delta_{p} \varphi=0 & \text { in } B_{\sqrt{\kappa} r} \backslash B_{\kappa r} \\ \varphi=0 & \text { on } \partial B_{\kappa r} \\ \varphi=1 & \text { on } \partial B_{\sqrt{\kappa} r}\end{cases}
$$

defined as 0 in $B_{\kappa r}$. Now we define

$$
v^{\prime}:=\varepsilon \sqrt{\kappa} r \varphi
$$

Note that $v^{\prime} \geq v$ on $\partial B_{\sqrt{\kappa} r}$. Finally, we define

$$
w:=\min \left(v, v^{\prime}\right) \text { in } B_{\sqrt{\kappa} r}, \quad w:=v \text { in } B_{\sqrt{\kappa} r}^{c},
$$

and we use $w$ as a comparison function in (4.2). We notice that $\{w=0\} \supset\{v=0\}$ and so from minimality of $v$ we conclude

$$
\int_{B_{\sqrt{\kappa} r}}|\nabla v|^{p} d x+\frac{\hat{\eta}}{2}|\{w=0\} \backslash\{v=0\}| \leq \int_{B_{\sqrt{\kappa} r}}|\nabla w|^{p} d x=\int_{B_{\sqrt{\kappa} r} \backslash B_{\kappa} r}|\nabla w|^{p} d x .
$$

Now we use the definition of $w$, positivity of $v^{\prime}$ in $B_{\sqrt{\kappa} r} \backslash B_{\kappa r}$, and convexity of $t \rightarrow t^{p}$ to get

$$
\begin{aligned}
& \int_{B_{\kappa r}}|\nabla v|^{p} d x+\frac{\hat{\eta}}{2}\left|B_{\kappa r} \cap\{v>0\}\right| \leq \int_{B_{\sqrt{\kappa} r} \backslash B_{\kappa r}}\left(|\nabla w|^{p}-|\nabla v|^{p}\right) d x \\
\leq & p \int_{B_{\sqrt{\kappa} r} \backslash B_{\kappa r}}|\nabla w|^{p-2} \nabla w \cdot \nabla(w-v) d x=p \int_{\partial B_{\kappa r}}\left|\nabla v^{\prime}\right|^{p-2} v \nabla v^{\prime} \cdot \nu .
\end{aligned}
$$

From the definition of $v^{\prime}$ we have

$$
\left|\nabla v^{\prime}\right| \leq C \frac{\varepsilon \sqrt{\kappa} r}{\kappa r-\sqrt{\kappa} r} \leq C \varepsilon
$$

So we have

$$
\int_{B_{\kappa r}}|\nabla v|^{p} d x+\frac{\hat{\eta}}{2}\left|B_{\kappa r} \cap\{v>0\}\right| \leq C \varepsilon^{p-1} \int_{\partial B_{\kappa r}} v .
$$

On the other hand, by trace inequality and Young inequality, and remembering the definition of $\varepsilon$, we can get

$$
\begin{aligned}
\int_{\partial B_{\kappa r}} v & \leq C\left(\frac{1}{r} \int_{B_{\kappa r}} v d x+\int_{B_{\kappa r}}|\nabla v| d x\right) \\
& \leq C\left(\sqrt{\kappa} \varepsilon\left|B_{\kappa r} \cap\{v>0\}\right|+\frac{1}{p} \int_{B_{\kappa r}}|\nabla v|^{p} d x+\frac{p-1}{p}\left|B_{\kappa r} \cap\{v>0\}\right|\right) \\
& \leq C(1+\varepsilon)\left(\int_{B_{\kappa r}}|\nabla v|^{p} d x+\left|B_{\kappa r} \cap\{v>0\}\right|\right) .
\end{aligned}
$$

Bringing it all together, we get

$\int_{B_{\kappa r}}|\nabla v|^{p} d x+\frac{\hat{\eta}}{2}\left|B_{\kappa r} \cap\{v>0\}\right| \leq C \varepsilon^{p-1}(1+\varepsilon)\left(\int_{B_{\kappa r}}|\nabla v|^{p} d x+\left|B_{\kappa r} \cap\{v>0\}\right|\right)$.

It remains to choose $c$ from the statement of the lemma small enough for $C \varepsilon^{p-1}(1+\varepsilon)$ to be smaller that $\min \left\{\frac{1}{2}, \frac{\hat{\eta}}{4}\right\}$. 
As in Section 4 of [DP05] these two lemmas imply Lipschitz continuity of minimizers and density estimates on the boundary of minimizing domains.

Lemma 5.9. Let $v_{j}$ be as above, $\Omega_{j}=\left\{v_{j}=0\right\}$. Then $\Omega_{j}$ is open and there exist constants $C=C(N, R), \rho_{0}=\rho_{0}(N, R)>0$ such that

(i) for every $x \in B_{R}$

$$
\frac{1}{C} \operatorname{dist}\left(x, \Omega_{j}\right) \leq v_{j} \leq C \operatorname{dist}\left(x, \Omega_{j}\right)
$$

(ii) $v_{j}$ are equi-Lipschitz;

(iii) for every $x \in \partial \Omega_{j}$ and $r \leq \rho_{0}$

$$
\frac{1}{C} \leq \frac{\left|\Omega_{j} \cap B_{r}(x)\right|}{\left|B_{r}(x)\right|} \leq\left(1-\frac{1}{C}\right) .
$$

Applying [DP05, Theorem 5.1] to $v_{j}$ (for more details on the proof see [AC81, Theorem 4.5]) we also have the following lemma.

Lemma 5.10. Let $v_{j}$ be as above, then there exists a Borel function $q_{u_{j}}$ such that

$$
\operatorname{div}\left(\left|\nabla v_{j}\right|^{p-2} \nabla v_{j}\right)=q_{v_{j}} \mathcal{H}^{N-1}\left\llcorner\partial^{*} \Omega_{j} .\right.
$$

Moreover, $0<c \leq-q_{v_{j}} \leq C, c=c(n, R), C=C(n, R)$ and $\mathcal{H}^{N-1}\left(\partial \Omega_{j} \backslash \partial^{*} \Omega_{j}\right)=0$.

Since $\Omega_{j}$ converge to $B_{1}$ in $L^{1}$ by Lemma 4.3 , the density estimates also give us the following convergence of boundaries.

Lemma 5.11. Let $\Omega_{j}$ be minimizers of (4.2). Then every limit point of $\Omega_{j}$ with respect to $L^{1}$ convergence is the unit ball centered at some $x_{\infty} \in B_{R}$. Moreover, the convergence holds also in the Kuratowski sense.

Corollary 5.12. In the setting of Lemma 5.11, for every $\delta>0$ there exists $j_{\delta}$ such that for $j \geq j_{\delta}$

for some $x_{j} \in B_{R}$.

$$
B_{1-\delta}\left(x_{j}\right) \subset \Omega_{j} \subset B_{1+\delta}\left(x_{j}\right)
$$

5.2. Higher regularity of the free boundary. To address the higher regularity of $\partial \Omega_{j}$, we are first going to prove that $q_{v_{j}}$ is smooth and then use the free boundary regularity result of [DP05]. Note that in order to apply [DP05, Theorem 9.1] we need to know that $\partial \Omega_{j}$ is flat, which follows from closeness of sets $\Omega_{j}$ to the ball.

We show smoothness of $q_{v_{j}}$ by using the Euler-Lagrange equations for our minimizing problem. We defined $\Omega_{j}$ in such a way that the following minimizing property holds

$$
\begin{aligned}
& \int_{\mathbb{R}^{N}}\left|\nabla v_{j}\right|^{p} d x+f_{\hat{\eta}}\left(\left|\left\{v_{j}=0\right\}\right|\right)+\sqrt{\varepsilon_{j}^{2}+\sigma^{2}\left(\alpha\left(\left\{v_{j}=0\right\}\right)-\varepsilon_{j}\right)^{2}} \\
& \leq \int_{\mathbb{R}^{N}}|\nabla v|^{p} d x+f_{\hat{\eta}}(|\{v=0\}|)+\sqrt{\varepsilon_{j}^{2}+\sigma^{2}\left(\alpha(\{v=0\})-\varepsilon_{j}\right)^{2}}
\end{aligned}
$$

for any $v \in W^{1,2}\left(\mathbb{R}^{N}\right)$ such that $0 \leq v \leq 1,\{v=0\} \subset B_{R}$.

To write Euler-Lagrange equations for $v_{j}$, we need to have (5.8) for $v_{j} \circ \Phi$ where $\Phi$ is a diffeomorphism of $\mathbb{R}^{N}$ close to the identity. Note that to make sure that 
$\left\{v_{j} \circ \Phi=0\right\}$ is contained in $B_{R}$ one needs to know that $\operatorname{dist}\left(\left\{v_{j}=0\right\}, \partial B_{R}\right)>0$. This follows from Corollary 5.12, up to translating $\Omega_{j}$. More precisely we will get the following optimality condition

$$
(p-1) q_{v_{j}}^{p}-\frac{\sigma^{2}\left(\alpha\left(\Omega_{j}\right)-\varepsilon_{j}\right)}{\sqrt{\varepsilon_{j}^{2}+\sigma^{2}\left(\alpha\left(\Omega_{j}\right)-\varepsilon_{j}\right)^{2}}}\left(\left|x-x_{\Omega_{j}}\right|-\left(f_{\Omega_{j}} \frac{y-x_{\Omega_{j}}}{\left|y-x_{\Omega_{j}}\right|} d y\right) \cdot x\right)=\Lambda_{j}
$$

for some constant $\Lambda_{j}>0$. This equation is an immediate consequence of the following lemma whose proof is almost the same as [BDPV15, Lemma 4.15] (which in turn is based on [AAC86]). For this reason we only highlight the most relevant changes, referring the reader to [BDPV15, Lemma 4.15] for more details.

Lemma 5.13. There exists $j_{0}$ such that for any $j \geq j_{0}$ and any two points $x_{1}$ and $x_{2}$ in the reduced boundary of $\Omega_{j}$ the following equality holds:

$$
\begin{aligned}
& (p-1) q_{v_{j}}^{p}\left(x_{1}\right)-\frac{\sigma^{2}\left(\alpha\left(\Omega_{j}\right)-\varepsilon_{j}\right)}{\sqrt{\varepsilon_{j}^{2}+\sigma^{2}\left(\alpha\left(\Omega_{j}\right)-\varepsilon_{j}\right)^{2}}}\left(\left|x_{1}-x_{\Omega_{j}}\right|-\left(f_{\Omega_{j}} \frac{y-x_{\Omega_{j}}}{\left|y-x_{\Omega_{j}}\right|} d y\right) \cdot x_{1}\right) \\
& =(p-1) q_{v_{j}}^{p}\left(x_{2}\right)-\frac{\sigma^{2}\left(\alpha\left(\Omega_{j}\right)-\varepsilon_{j}\right)}{\sqrt{\varepsilon_{j}^{2}+\sigma^{2}\left(\alpha\left(\Omega_{j}\right)-\varepsilon_{j}\right)^{2}}}\left(\left|x_{2}-x_{\Omega_{j}}\right|-\left(f_{\Omega_{j}} \frac{y-x_{\Omega_{j}}}{\left|y-x_{\Omega_{j}}\right|} d y\right) \cdot x_{2}\right) .
\end{aligned}
$$

Proof. We argue by contradiction. Assume there exist $x_{1}, x_{2} \in \partial^{*}\left\{v_{j}=0\right\}$ such that

$$
\begin{aligned}
& (p-1) q_{v_{j}}^{p-1}\left(x_{1}\right)-\frac{\sigma^{2}\left(\alpha\left(\Omega_{j}\right)-\varepsilon_{j}\right)}{\sqrt{\varepsilon_{j}^{2}+\sigma^{2}\left(\alpha\left(\Omega_{j}\right)-\varepsilon_{j}\right)^{2}}}\left(\left|x_{1}-x_{\Omega_{j}}\right|-\left(f_{\Omega_{j}} \frac{y-x_{\Omega_{j}}}{\left|y-x_{\Omega_{j}}\right|} d y\right) \cdot x_{1}\right) \\
& <(p-1) q_{v_{j}}^{p-1}\left(x_{2}\right)-\frac{\sigma^{2}\left(\alpha\left(\Omega_{j}\right)-\varepsilon_{j}\right)}{\sqrt{\varepsilon_{j}^{2}+\sigma^{2}\left(\alpha\left(\Omega_{j}\right)-\varepsilon_{j}\right)^{2}}}\left(\left|x_{2}-x_{\Omega_{j}}\right|-\left(f_{\Omega_{j}} \frac{y-x_{\Omega_{j}}}{\left|y-x_{\Omega_{j}}\right|} d y\right) \cdot x_{2}\right) .
\end{aligned}
$$

Using this inequality, we are going to construct a variation contradicting (5.8). We take a smooth radial symmetric function $\phi(x)=\phi(|x|)$ supported in $B_{1}$ and define the following diffeomorphism for small $\tau$ and $\rho$ :

$$
\Phi_{\tau}^{\rho}(x)= \begin{cases}x+\tau \rho \phi\left(\left|\frac{x-x_{1}}{\rho}\right|\right) \nu\left(x_{1}\right), & x \in B_{\rho}\left(x_{1}\right), \\ x-\tau \rho \phi\left(\left|\frac{x-x_{2}}{\rho}\right|\right) \nu\left(x_{2}\right), & x \in B_{\rho}\left(x_{2}\right), \\ x, & \text { otherwise. }\end{cases}
$$

We define the function

$$
v_{\tau}^{\rho}:=v \circ\left(\Phi_{\tau}^{\rho}\right)^{-1}
$$

and we define a competitor domain $\Omega_{\tau}^{\rho}$ as follows:

$$
\Omega_{\tau}^{\rho}:=\left\{v_{\tau}^{\rho}=0\right\} .
$$

Note that $1-v_{\tau}^{\rho}$ is a competitor for the $p$-capacity of $\Omega_{\tau}^{\rho}$, so we have

$$
\operatorname{cap}_{p}\left(\Omega_{\tau}^{\rho}\right) \leq \int_{\left(\Omega_{\tau}^{\rho}\right)^{c}}\left|\nabla v_{\tau}^{\rho}\right|^{p} .
$$


Now we are going to show that for $\tau$ and $\rho$ small enough $\mathscr{C}_{\hat{\eta}}\left(\Omega_{\tau}^{\rho}\right)<\mathscr{C}_{\hat{\eta}}(\Omega)$. To do that, we first compute the variation of all the terms involved in $\mathscr{C}_{\hat{\eta}}$.

Volume. By arguing as in [BDPV15, Lemma 4.15] one gets

$$
\begin{aligned}
\left|\Omega_{\tau}^{\rho}\right|-|\Omega| & =\tau \rho^{N}\left(\int_{\left\{y \cdot \nu\left(x_{1}\right)=0\right\} \cap B_{1}} \phi(|y|)-\int_{\left\{y \cdot \nu\left(x_{2}\right)=0\right\} \cap B_{1}} \phi(|y|)\right)+o(\tau) \rho^{N}+o_{\tau}\left(\rho^{N}\right) \\
& =o(\tau) \rho^{N}+o_{\tau}\left(\rho^{N}\right),
\end{aligned}
$$

where $o_{\tau}\left(\rho^{N}\right) \rho^{-N}$ goes to zero as $\rho \rightarrow 0$ and $o(\tau)$ is independent on $\rho$.

Barycenter. Assume that that $x_{\Omega}=0$, as in [BDPV15, Lemma 4.15] one gets,

$$
x_{\Omega_{\tau}^{\rho}}=-\rho^{N} \tau \frac{x_{1}-x_{2}}{|\Omega|}\left(\int_{\left\{y_{1}=0\right\} \cap B_{1}} \phi(|y|)\right)+\rho^{N} o(\tau)+o_{\tau}\left(\rho^{N}\right) .
$$

Asymmetry. Again by the very same computations as in [BDPV15, Lemma 4.15] one gets

$$
\begin{array}{r}
\alpha\left(\Omega_{\tau}^{\rho}\right)-\alpha(\Omega)=-\rho^{N} \tau\left(\int_{\left\{y_{1}=0\right\} \cap B_{1}} \phi(|y|)\right)\left(\left|x_{1}\right|-\left|x_{2}\right|+\left(f_{\Omega} \frac{y}{|y|} d y\right) \cdot\left(x_{1}-x_{2}\right)\right) \\
+o(\tau) \rho^{N}+o_{\tau}\left(\rho^{N}\right) .
\end{array}
$$

Dirichlet energy. Here one can argue as in [FZ16, Lemma 3.19] to get

$$
\begin{aligned}
\operatorname{cap}_{p}\left(\Omega_{\tau}^{\rho}\right)-\operatorname{cap}_{p}(\Omega) \leq \tau \rho^{N}(p-1)\left(\left|q\left(x_{1}\right)\right|^{p}-\left|q\left(x_{2}\right)\right|^{p}\right) & \int_{B_{1} \cap\left\{y_{1}=0\right\}} \phi(|y|) d y \\
& +o(\tau) \rho^{N}+o_{\tau}\left(\rho^{N}\right) .
\end{aligned}
$$

Combining the above estimates one gets

$$
\begin{aligned}
& \left(\int_{B_{1} \cap\left\{y_{1}=0\right\}} \phi(|y|) d y\right)^{-1} \frac{\mathscr{C}_{\hat{\eta}, j}\left(\Omega_{\tau}^{\rho}\right)-\mathscr{C}_{\hat{\eta}, j}(\Omega)}{\rho^{N}}=\tau\left((p-1)\left(\left|q\left(x_{1}\right)\right|^{p}-\left|q\left(x_{2}\right)\right|^{p}\right)\right) \\
& -\tau \frac{\sigma^{2}\left(\alpha(\Omega)-\varepsilon_{j}\right)}{\sqrt{\varepsilon_{j}^{2}+\sigma^{2}\left(\alpha(\Omega)-\varepsilon_{j}\right)^{2}}}\left(\left|x_{1}\right|-\left|x_{2}\right|+\left(f_{\Omega} \frac{y}{|y|} d y\right) \cdot\left(x_{1}-x_{2}\right)\right)+o(\tau)+o_{\tau}(1) .
\end{aligned}
$$

According to (5.9) the quantity in parentheses is strictly negative. Thus, we get a contradiction with the minimality of $\Omega$ for $\rho$ and $\tau$ small enough.

Lemma 5.14 (Smoothness of $q_{v}$ ). There exist constants $\delta=\delta(N, R)>0, j_{0}=$ $j_{0}(N, R), \sigma_{0}=\sigma_{0}(N, R)>0$ such that for every $j \geq j_{0}, \sigma \leq \sigma_{0}$ the functions $q_{v_{j}}$ belong to $C^{\infty}\left(\mathcal{N}_{\delta}\left(\partial \Omega_{j}\right)\right)$.

Moreover, for every $k$ there exists a constant $C=C(k, N, R)$ such that

$$
\left\|q_{v_{j}}\right\|_{C^{k}\left(\mathcal{N}_{\delta}\left(\partial \Omega_{j}\right)\right)} \leq C
$$

for every $j \geq j_{0}$. 
Proof. We would like to write an explicit formula for $q_{v_{j}}$ using Euler-Lagrange equations, namely

$$
q_{v_{j}}=-\left(\frac{\sigma^{2}\left(\alpha\left(\Omega_{j}\right)-\varepsilon_{j}\right)}{\sqrt{\varepsilon_{j}^{2}+\sigma^{2}\left(\alpha\left(\Omega_{j}\right)-\varepsilon_{j}\right)^{2}}}\left(\left|x-x_{\Omega_{j}}\right|-\left(f_{\Omega_{j}} \frac{y-x_{\Omega_{j}}}{\left|y-x_{\Omega_{j}}\right|} d y\right) \cdot x\right)+\Lambda_{j}\right)^{\frac{1}{p}} .
$$

To do that, we need to show that the quantity in the parenthesis is bounded away from zero. Indeed, $q_{v_{j}}$ is bounded from above and below independently of $j$ and

$$
\left|\frac{\sigma^{2}\left(\alpha\left(\Omega_{j}\right)-\varepsilon_{j}\right)}{\sqrt{\varepsilon_{j}^{2}+\sigma^{2}\left(\alpha\left(\Omega_{j}\right)-\varepsilon_{j}\right)^{2}}}\left(\left|x-x_{\Omega_{j}}\right|-\left(f_{\Omega_{j}} \frac{y-x_{\Omega_{j}}}{\left|y-x_{\Omega_{j}}\right|} d y\right) \cdot x\right)\right| \leq C(N, R) \sigma .
$$

Then it follows from the Euler-Lagrange equations that also $\Lambda_{j}$ is bounded from above and below independently of $j$. Thus, for $\sigma$ small enough we can write the above-mentioned explicit formula for $q_{v_{j}}$ and get the conclusion of the lemma.

Now we want to apply the results of [DP05]. We can't apply them directly, since the equation there is slightly different. More precisely, in [DP05] the authors are considering solutions of the equation

$$
\operatorname{div}\left(|\nabla u|^{p-2} \nabla u\right)=\mathcal{H}^{n-1}\llcorner\partial\{u>0\}
$$

whereas $v_{j}$ satisfies

$$
\operatorname{div}\left(\left|\nabla v_{j}\right|^{p-2} \nabla v_{j}\right)=q_{v_{j}} \mathcal{H}^{n-1}\left\llcorner\partial\left\{v_{j}>0\right\} .\right.
$$

However, since $q_{v_{j}}$ is smooth, the proof works in exactly the same way (see also Appendix of [FZ16] for the same result for a slightly different equation, the proof becomes more involved in that case). The idea is that flatness improves in smaller balls if the free boundary is sufficiently flat in some ball.

First, we need to recall the definition of flatness for the free boundary, see [AC81, Definition 7.1] (here it is applied to $v$ ).

Definition 5.15. Let $\mu_{-}, \mu_{+} \in(0,1]$. A weak solution $v$ of $(5.7)$ is said to be of class $F\left(\mu_{-}, \mu_{+}, \infty\right)$ in $B_{\rho}\left(x_{0}\right)$ in a direction $\nu \in S^{N-1}$ if $x_{0} \in \partial\{v=0\}$ and

$$
\begin{cases}v(x)=0 & \text { for }\left(x-x_{0}\right) \cdot \nu \leq-\mu_{-} \rho, \\ v(x) \geq q_{v}\left(x_{0}\right)\left(\left(x-x_{0}\right) \cdot \nu-\mu_{+} \rho\right) & \text { for }\left(x-x_{0}\right) \cdot \nu \geq \mu_{+} \rho .\end{cases}
$$

See also Figure 1.

Remark 5.16. In particular, if the free boundary is sufficiently flat near $x_{0}$, that is, it is contained in a thin strip, then the corresponding $v$ is of class $F(\mu, 1, \infty)$ in a small ball around $x_{0}$ for some $\mu$.

We are going to use that flat free boundaries are smooth. The following theorem is a slight generalization of [DP05, Theorem 9.1] and we omit the proof since it is almost identical.

Theorem 5.17. Let $u$ be a weak solution of (5.7) and assume that $q_{v}$ is Lipschitz continuous. There are constants $\gamma, \mu_{0}, \kappa, C$ such that if $v$ is of class $F(\mu, 1, \infty)$ in 


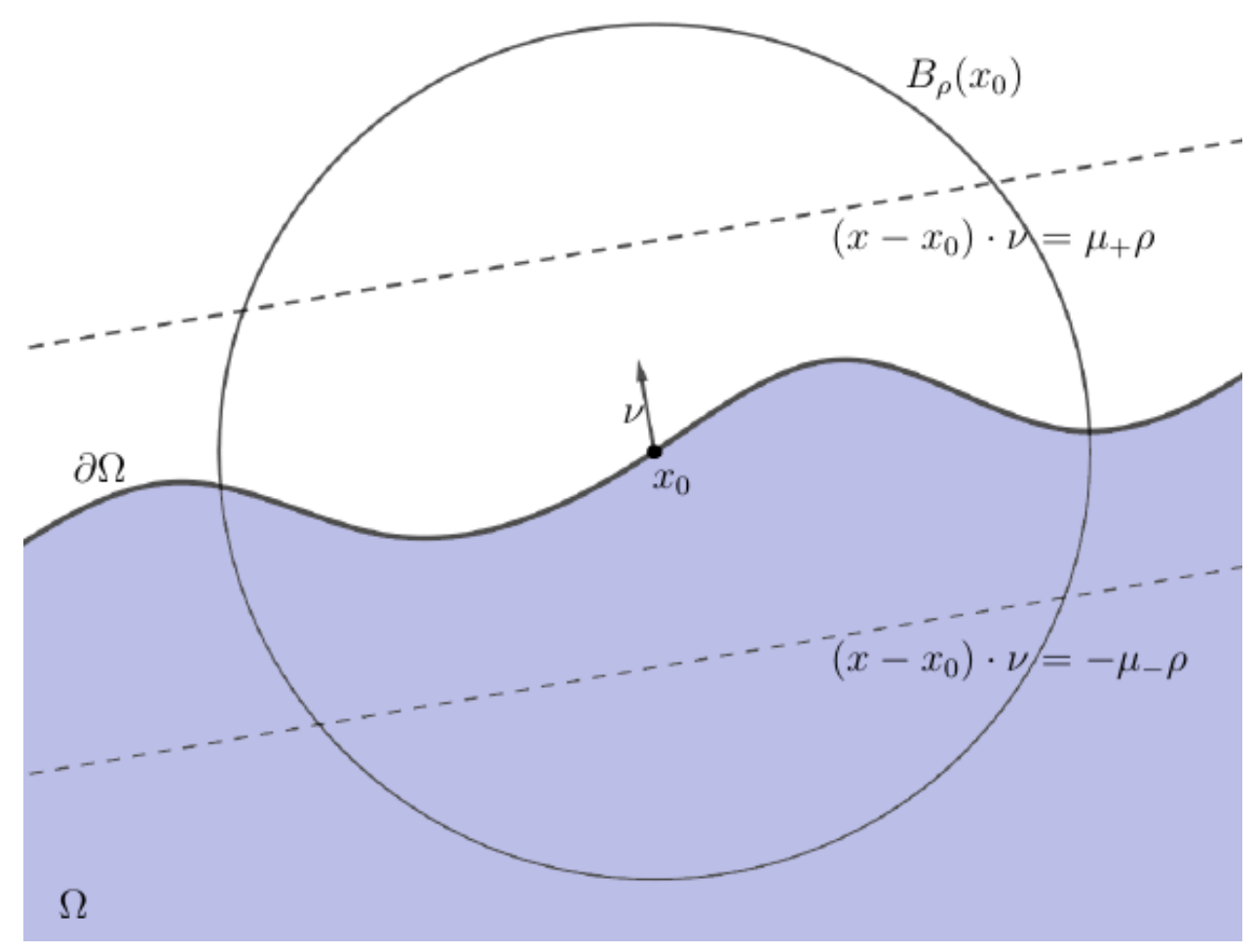

Figure 1. $v$ of the class $F\left(\mu_{-}, \mu_{+}, \infty\right)$ in $B_{\rho}\left(x_{0}\right)$ in a direction $\nu \in S^{N-1}$

$B_{4 \rho}\left(x_{0}\right)$ in some direction $\nu \in S^{N-1}$ with $\mu \leq \mu_{0}$ and $\rho \leq \kappa \mu^{2}$, then there exists a $C^{1, \gamma}$ function $f: \mathbb{R}^{N-1} \rightarrow \mathbb{R}$ with $\|f\|_{C^{1, \gamma}} \leq C \mu$ such that

$$
\partial\{v=0\} \cap B_{\rho}\left(x_{0}\right)=\left(x_{0}+\operatorname{graph}_{\nu} f\right) \cap B_{\rho}\left(x_{0}\right),
$$

where $\left.\operatorname{graph}_{\nu} f=\left\{x \in \mathbb{R}^{N}: x \cdot \nu=f(x-x \cdot \nu) \nu\right)\right\}$.

Moreover if $q_{v} \in C^{k, \gamma}$ in some neighborhood of $\left\{u_{j}=1\right\}$, then $f \in C^{k+1, \gamma}$ and $\|f\|_{C^{k+1, \gamma}} \leq C\left(N, R,\left\|q_{v}\right\|_{C^{k, \gamma}}\right)$.

Proof of Theorem 3.2. We define $\Omega_{j}$ as minimizers of (4.2). To get the desired sequence we will rescale the sets $\left\{\Omega_{j}\right\}$ so that they have the correct volume. We need to show that $\left\{\Omega_{j}\right\}$ converges smoothly to the ball $B_{1}$. Indeed one then define

$$
U_{j}=\lambda_{j}\left(\Omega_{j}-x_{\Omega_{j}}\right) .
$$

Lemma 4.3 then implies all the desired properties of $U_{j}$, compare with [BDPV15, Proof of Proposition 4.4].

Let $\mu_{0}, \kappa$ be as in Theorem 5.17 and $\mu<\mu_{0}$ to be fixed later. Let $\bar{x}$ be some point on the boundary of $B_{1}$. As $\partial B_{1}$ is smooth, it lies inside a narrow strip in the neighborhood of $\bar{x}$. More precisely, there exists $\rho_{0}=\rho_{0}(\mu) \leq \kappa \mu^{2}$ such that for every $\rho<\rho_{0}$ and every $\bar{x} \in \partial B_{1}$

$$
\partial B_{1} \cap B_{5 \rho}(\bar{x}) \subset\left\{x:\left|(x-\bar{x}) \cdot \nu_{\bar{x}}\right| \leq \mu \rho\right\} .
$$


We know that $\partial \Omega_{j}$ are converging to $\partial B_{1}$ in the sense of Kuratowski. Thus, there exists a point $x_{0} \in \partial \Omega_{j} \cap B_{\mu \rho_{0}}(\bar{x})$ such that

$$
\partial \Omega_{j} \cap B_{4 \rho_{0}}\left(x_{0}\right) \subset\left\{x:\left|\left(x-x_{0}\right) \cdot \nu_{\bar{x}}\right| \leq 4 \mu \rho_{0}\right\} .
$$

So, $u_{j}$ is of class $F(\mu, 1, \infty)$ in $B_{4 \rho_{0}}\left(x_{0}\right)$ with respect to the direction $\nu_{\bar{x}}$ and by Theorem 5.17, $\partial \Omega_{j} \cap B_{\rho_{0}}\left(x_{0}\right)$ is the graph of a smooth function with respect to $\nu_{\bar{x}}$. More precisely, for $\mu$ small enough there exists a family of smooth functions $g_{j}^{\bar{x}}$ with uniformly bounded $C^{k}$ norms such that

$$
\partial \Omega_{j} \cap B_{\rho_{0}}(\bar{x})=\left\{x+g_{j}^{\bar{x}}(x) x: x \in \partial B_{1}\right\} \cap B_{\rho_{0}}(\bar{x}) .
$$

By a covering argument this gives a family of smooth functions $g_{j}$ with uniformly bounded $C^{k}$ norms such that

$$
\partial \Omega_{j}=\left\{x+g_{j}(x) x: x \in \partial B_{1}\right\} .
$$

By Ascoli-Arzelà and convergence to $\partial B_{1}$ in the sense of Kuratowski, we get that $g_{j} \rightarrow 0$ in $C^{k-1}\left(\partial B_{1}\right)$, hence the smooth convergence of $\partial \Omega_{j}$.

\section{Reduction to Bounded sets}

To complete the proof of Theorem 1.4 one needs to show that one can consider only sets with uniformly bounded diameter. To this end let us introduce the following.

Definition 6.1. Let $\Omega$ be an open set in $\mathbb{R}^{n}$ with $|\Omega|=\left|B_{1}\right|$. Then we define the deficit of $\Omega$ as the difference between its $p$-capacity and the $p$-capacity of the unit ball:

$$
D(\Omega)=\operatorname{cap}_{p}(\Omega)-\operatorname{cap}_{p}\left(B_{1}\right) .
$$

Here is the key lemma for reducing Theorem 1.4 to Theorem 3.1.

Lemma 6.2. There exist constants $C=C(N), \delta=\delta(N)>0$ and $d=d(N)$ such that for any $\Omega \subset \mathbb{R}^{n}$ open with $|\Omega|=\left|B_{1}\right|$ and $D(\Omega) \leq \delta$, we can find a new set $\tilde{\Omega}$ enjoying the following properties

(1) $\operatorname{diam}(\tilde{\Omega}) \leq d$,

(2) $|\tilde{\Omega}|=\left|B_{1}\right|$,

(3) $D(\tilde{\Omega}) \leq C D(\Omega)$,

(4) $\mathcal{A}(\tilde{\Omega}) \geq \mathcal{A}(\Omega)-C D(\Omega)$.

We are going to define $\tilde{\Omega}$ as a suitable dilation of $\Omega \cap B_{S}$ for some large $S$. Hence, we first show the following estimates on the $p$-capacity of $\Omega \cap B_{S}$.

Lemma 6.3. Let $S^{\prime}>S$. Then there exists a constant $c=c\left(S^{\prime}\right)$ such that for any open set $\Omega \subset \mathbb{R}^{N}$ with $|\Omega|=\left|B_{1}\right|$ the following inequalities hold:

$\operatorname{cap}_{p}\left(B_{1}\right)\left(1-\frac{\left|\Omega \backslash B_{S}\right|}{\left|B_{1}\right|}\right)^{\frac{N-p}{N}} \leq \operatorname{cap}_{p}\left(\Omega \cap B_{S}\right) \leq \operatorname{cap}(\Omega)-c\left(1-\frac{S}{S^{\prime}}\right)^{p}\left|\Omega \backslash B_{S^{\prime}}\right|^{\frac{N-p}{N}}$.

Proof of Lemma 6.3. The first inequality is a direct consequence of the classical isocapacitary inequality. To prove the second one we are going to use the estimates 
for the capacitary potential of $B_{S}$ for which the exact formula can be written. Denote by $u_{\Omega}$ and $u_{S}$ the capacitary potentials of $\Omega$ and $\Omega \cap B_{S}$ respectively. We first write $\operatorname{cap}_{p}\left(\Omega \cap B_{S}\right)=\operatorname{cap}_{p}(\Omega)+\int_{\mathbb{R}^{n}}\left|\nabla u_{S}\right|^{p}-\left|\nabla u_{\Omega}\right|^{p}=\operatorname{cap}_{p}(\Omega)-\int_{\left(\Omega \cap B_{S}\right)^{c}}\left(\left|\nabla u_{\Omega}\right|^{p}-\left|\nabla u_{S}\right|^{p}\right)$.

Let us show that

$$
\int_{\left(\Omega \cap B_{S}\right)^{c}}\left|\nabla u_{\Omega}\right|^{p}-\left|\nabla u_{S}\right|^{p} \geq c(p) \int_{\Omega \backslash B_{S}}\left|\nabla u_{S}\right|^{p} .
$$

We first claim that

$$
\int_{\left(\Omega \cap B_{S}\right)^{c}}\left|\nabla u_{S}\right|^{p-2} \nabla u_{S} \cdot \nabla\left(u_{\Omega}-u_{S}\right) \geq 0 .
$$

Note that (6.2) becomes equality for a smooth domain $\Omega$. We need to be more careful for an arbitrary domain however. Since $\Omega \cap B_{S} \subset \Omega$, for any $\varepsilon \geq 0$ the function $u_{S}^{\varepsilon}:=(1-\varepsilon) u_{S}+\varepsilon u_{\Omega}$ is a competitor for the $p$-capacity of $\Omega \cap B_{S}$. Thus,

$$
\begin{aligned}
0 & \geq \int_{\left(\Omega \cap B_{S}\right)^{c}}\left|\nabla u_{S}\right|^{p}-\int_{\left(\Omega \cap B_{S}\right)^{c}}\left|\nabla u_{S}^{\varepsilon}\right|^{p} \geq p \int_{\left(\Omega \cap B_{S}\right)^{c}}\left|\nabla u_{S}^{\varepsilon}\right|^{p-2} \nabla u_{S}^{\varepsilon} \cdot \nabla\left(u_{S}-u_{S}^{\varepsilon}\right) \\
& =p \varepsilon \int_{\left(\Omega \cap B_{S}\right)^{c}}\left|(1-\varepsilon) \nabla u_{S}+\varepsilon \nabla u_{\Omega}\right|^{p-2}\left((1-\varepsilon) \nabla u_{S}+\varepsilon \nabla u_{\Omega}\right) \cdot \nabla\left(u_{S}-u_{\Omega}\right) .
\end{aligned}
$$

We consider separately two cases. If $1<p<2$, by concavity of $t \rightarrow t^{p-2}$ we have

$$
\begin{aligned}
0 & \geq \int_{\left(\Omega \cap B_{S}\right)^{c}}\left|(1-\varepsilon) \nabla u_{S}+\varepsilon \nabla u_{\Omega}\right|^{p-2}\left((1-\varepsilon) \nabla u_{S}+\varepsilon \nabla u_{\Omega}\right) \cdot \nabla\left(u_{S}-u_{\Omega}\right) \\
& \geq \int_{\left(\Omega \cap B_{S}\right)^{c}}\left((1-\varepsilon)\left|\nabla u_{S}\right|^{p-2}+\varepsilon\left|\nabla u_{\Omega}\right|^{p-2}\right)\left((1-\varepsilon) \nabla u_{S}+\varepsilon \nabla u_{\Omega}\right) \cdot \nabla\left(u_{S}-u_{\Omega}\right) \\
& =(1-\varepsilon)^{2} \int_{\left(\Omega \cap B_{S}\right)^{c}}\left|\nabla u_{S}\right|^{p-2} \nabla u_{S} \cdot \nabla\left(u_{S}-u_{\Omega}\right) \\
& +\varepsilon(1-\varepsilon) \int_{\left(\Omega \cap B_{S}\right)^{c}}\left|\nabla u_{\Omega}\right|^{p-2} \nabla u_{S} \cdot \nabla\left(u_{S}-u_{\Omega}\right)+\varepsilon^{2} \int_{\left(\Omega \cap B_{S}\right)^{c}}\left|\nabla u_{\Omega}\right|^{p-2} \nabla u_{\Omega} \cdot \nabla\left(u_{S}-u_{\Omega}\right) \\
& +\varepsilon^{2} \int_{\left(\Omega \cap B_{S}\right)^{c}}\left|\nabla u_{S}\right|^{p-2} \nabla u_{\Omega} \cdot \nabla\left(u_{S}-u_{\Omega}\right) .
\end{aligned}
$$

We know that the integrals in the last equation are finite since both $u_{\Omega}$ and $u_{S}$ are in $D^{1, p}$. Thus, sending $\varepsilon$ to 0 we get the inequality (6.2) in the case $1<p<2$. As for the case $p \geq 2$, we use that $|a+b|^{p-2} \geq c(p)|a|^{p-2}-C(p)|b|^{p-2}$ for some positive constants $c$ and $C$ and hence

$$
\begin{aligned}
0 & \geq \int_{\left(\Omega \cap B_{S}\right)^{c}}\left|(1-\varepsilon) \nabla u_{S}+\varepsilon \nabla u_{\Omega}\right|^{p-2}\left((1-\varepsilon) \nabla u_{S}+\varepsilon \nabla u_{\Omega}\right) \cdot \nabla\left(u_{S}-u_{\Omega}\right) \\
& \geq \int_{\left(\Omega \cap B_{S}\right)^{c}}\left(c(p)(1-\varepsilon)^{p-2}\left|\nabla u_{S}\right|^{p-2}-C(p) \varepsilon^{p-2}\left|\nabla u_{\Omega}\right|^{p-2}\right)\left((1-\varepsilon) \nabla u_{S}+\varepsilon \nabla u_{\Omega}\right) \cdot \nabla\left(u_{S}-u_{\Omega}\right) .
\end{aligned}
$$

As for the previous case, we can now send $\varepsilon$ to 0 and get the inequality (6.2) for $p \geq 2$. 
Now we are ready to prove (6.1). We consider two cases: $p \geq 2$ and $1<p<2$. For both we will be using an inequality of Lemma A.2. For $p \geq 2$ we have

$$
\begin{aligned}
& \int_{\left(\Omega \cap B_{S}\right)^{c}}\left|\nabla u_{\Omega}\right|^{p}-\left|\nabla u_{S}\right|^{p} \geq c(p) \int_{\left(\Omega \cap B_{S}\right)^{c}}\left|\nabla\left(u_{\Omega}-u_{S}\right)\right|^{p} \\
& \quad+p \int_{\left(\Omega \cap B_{S}\right)^{c}}\left|\nabla u_{S}\right|^{p-2} \nabla u_{S} \cdot \nabla\left(u_{\Omega}-u_{S}\right) \\
& \quad \geq c(p) \int_{\left(\Omega \cap B_{S}\right)^{c}}\left|\nabla\left(u_{\Omega}-u_{S}\right)\right|^{p} \geq c(p) \int_{\Omega \backslash B_{S}}\left|\nabla\left(u_{\Omega}-u_{S}\right)\right|^{p} \\
& \quad=c(p) \int_{\Omega \backslash B_{S}}\left|\nabla u_{S}\right|^{p},
\end{aligned}
$$

where for the second inequality we used (6.2). As for the case $1<p<2$, we have

$$
\begin{array}{r}
\int_{\left(\Omega \cap B_{S}\right)^{c}}\left|\nabla u_{\Omega}\right|^{p}-\left|\nabla u_{S}\right|^{p} \geq c(p) \int_{\left(\Omega \cap B_{S}\right)^{c}}\left(\left|\nabla u_{\Omega}\right|^{2}+\left|\nabla u_{S}\right|^{2}\right)^{\frac{p-2}{2}}\left|\nabla\left(u_{\Omega}-u_{S}\right)\right|^{2} \\
+p \int_{\left(\Omega \cap B_{S}\right)^{c}}\left|\nabla u_{S}\right|^{p-2} \nabla u_{S} \cdot \nabla\left(u_{\Omega}-u_{S}\right) \\
\geq c(p) \int_{\left(\Omega \cap B_{S}\right)^{c}}\left(\left|\nabla u_{\Omega}\right|^{2}+\left|\nabla u_{S}\right|^{2}\right)^{\frac{p-2}{2}}\left|\nabla\left(u_{\Omega}-u_{S}\right)\right|^{2} \\
\geq c(p) \int_{\Omega \backslash B_{S}}\left(\left|\nabla u_{\Omega}\right|^{2}+\left|\nabla u_{S}\right|^{2}\right)^{\frac{p-2}{2}}\left|\nabla\left(u_{\Omega}-u_{S}\right)\right|^{2} \\
=c(p) \int_{\Omega \backslash B_{S}}\left|\nabla u_{S}\right|^{p},
\end{array}
$$

where for the last equality we used that $u_{\Omega} \equiv 1$ in $\Omega$.

We would like to show that $\int_{\Omega \backslash B_{S}}\left|\nabla u_{S}\right|^{p}$ cannot be too small. To this end let us set $v_{S}=1-u_{S}$. By Sobolev's embedding we get

$$
\int_{\Omega \backslash B_{S}}\left|\nabla u_{S}\right|^{p}=\int_{\Omega \backslash B_{S}}\left|\nabla v_{S}\right|^{p} \geq c(N)\left(\int_{\Omega \backslash B_{S}}\left|v_{S}\right|^{p^{*}}\right)^{\frac{p}{p^{*}}},
$$

where $p^{*}$ is the Sobolev exponent. Let us denote by $z_{S}$ the capacitary potential of $B_{S}$ :

$$
z_{S}=\left(1-\frac{S^{\frac{n-p}{p-1}}}{|x|^{\frac{n-p}{p-1}}}\right)_{+}
$$

By the maximum principle, $v_{S} \geq z_{S}$, hence

$$
\begin{aligned}
\int_{\Omega \backslash B_{S}}\left|v_{S}\right|^{p^{*}} & \geq \int_{\Omega \backslash B_{S}}\left|z_{S}\right|^{p^{*}} \\
& \geq \int_{\Omega \backslash B_{S^{\prime}}}\left|z_{S}\right|^{p^{*}} \geq\left(1-\left(\frac{S}{S^{\prime}}\right)^{\frac{n-p}{p-1}}\right)^{p^{*}}\left|\Omega \backslash B_{S^{\prime}}\right| .
\end{aligned}
$$


Hence

$$
\begin{aligned}
\operatorname{cap}_{p}\left(\Omega \cap B_{S}\right) & \leq \operatorname{cap}_{p}(\Omega)-c(N)\left(1-\left(\frac{S}{S^{\prime}}\right)^{\frac{n-p}{p-1}}\right)^{p}\left|\Omega \backslash B_{S^{\prime}}\right|^{\frac{N-p}{N}} \\
& \leq \operatorname{cap}_{p}(\Omega)-c\left(1-\frac{S}{S^{\prime}}\right)^{p}\left|\Omega \backslash B_{S^{\prime}}\right|^{\frac{N-p}{N}}
\end{aligned}
$$

concluding the proof.

We can now prove Lemma 6.2.

Proof of Lemma 6.2. The proof is almost identical to the proof of [DPMM19, Lemma 6.2]. We repeat it here for convenience of the reader.

Let us assume without loss of generality that the ball achieving the asymmetry of $\Omega$ is $B_{1}$. As was already mentioned, we are going to show that there exists an $\tilde{\Omega}$ of the form $\lambda\left(\Omega \cap B_{S}\right)$ for suitable $S$ and $\lambda$ satisfying all the desired properties. Let us set

$$
b_{k}:=\frac{\left|\Omega \backslash B_{2-2^{k}}\right|}{\left|B_{1}\right|} \leq 1 .
$$

Note that by Theorem 1.2 we can assume that $b_{1} \leq 2 \mathcal{A}(\Omega)$ is as small as we wish (independently on $\Omega$ up to choose $\delta$ sufficiently small. Lemma 6.3 gives

$\operatorname{cap}_{p}(\Omega)-c\left(\frac{2^{-(k+1)}}{2-2^{-(k+1)}}\right)^{p} b_{k+1}^{\frac{N-2}{N}} \geq \operatorname{cap}_{p}\left(B_{1}\right)\left(1-b_{k}\right)^{\frac{N-p}{N}} \geq \operatorname{cap}_{p}\left(B_{1}\right)-\operatorname{cap}_{p}\left(B_{1}\right) b_{k}$

which implies

$$
c b_{k+1} \leq\left(4^{N /(N-p)}\right)^{k}\left(D(\Omega)+C b_{k}\right)^{\frac{N}{N-p}} .
$$

We now claim that there exists $\bar{k}$ such that

$$
b_{\bar{k}} \leq D(\Omega) .
$$

Indeed, otherwise by (6.3) we would get

$$
b_{k+1} \leq C\left(4^{N /(N-p)}\right)^{k}\left(D(\Omega)+C b_{k}\right)^{\frac{N}{N-p}} \leq\left(4^{N /(N-p)}\right)^{k} C^{\prime} b_{k}^{\frac{N}{N-p}} \leq M^{k} b_{k}^{\frac{N}{N-p}}
$$

for all $k \in \mathbb{N}$, where $M=M(N)$. Iterating the last inequality, we obtain

$$
b_{k+1} \leq\left(M b_{1}\right)^{\left(\frac{N}{N-p}\right)^{k}} \underset{k \rightarrow \infty}{\longrightarrow} 0
$$

if $b_{1}$ is small enough, which by Theorem 1.2 we can assume up to choose $\delta=\delta(N) \ll$ 1 .

We define $\tilde{\Omega}$ as a properly rescaled intersection of $\Omega$ with a ball. Let $\bar{k}$ be such that $b_{\bar{k}} \leq D(\Omega)$

$$
\tilde{\Omega}:=\left(\frac{\left|B_{1}\right|}{\left|\Omega \cap B_{R}\right|}\right)^{\frac{1}{N}}\left(\Omega \cap B_{R}\right)=\left(1-b_{\bar{k}}\right)^{-\frac{1}{N}}\left(\Omega \cap B_{S}\right),
$$

where $S:=2-2^{-\bar{k}} \leq 2$. Note that $|\tilde{\Omega}|=\left|B_{1}\right|$. We now check all the remaining properties: 
- Bound on the diameter:

$$
\operatorname{diam}(\tilde{\Omega}) \leq 2 \cdot 2(1-D(\Omega))^{-\frac{1}{N}} \leq 4(1-\delta)^{-\frac{1}{N}} \leq 4 .
$$

up to choose $\delta=\delta(N) \ll 1$.

- Bound on the deficit:

$$
\begin{aligned}
D(\tilde{\Omega}) & =\operatorname{cap}_{p}(\tilde{\Omega})-\operatorname{cap}_{p}\left(B_{1}\right)=\operatorname{cap}_{p}\left(\Omega \cap B_{S}\right)\left(1-b_{\bar{K}}\right)^{-\frac{N-p}{N}}-\operatorname{cap}_{p}\left(B_{1}\right) \\
& \leq \operatorname{cap}_{p}(\Omega)\left(1-b_{\bar{k}}\right)^{-\frac{N-p}{N}}-\operatorname{cap}_{p}\left(B_{1}\right) \\
& \leq \operatorname{cap}_{p}(\Omega)-\operatorname{cap}_{p}\left(B_{1}\right)+\frac{2(N-p) \operatorname{cap}_{p}(\Omega)}{N} b_{\bar{k}} \leq C(N) D(\Omega) .
\end{aligned}
$$

since $b_{\bar{k}} \leq D(\Omega) \ll 1$ and, in particular, $\operatorname{cap}_{p}(\Omega) \leq 2 \operatorname{cap}_{p}\left(B_{1}\right)$.

- Bound on the asymmetry: Let $r:=\left(1-b_{\bar{k}}\right)^{-1} \in(1,2)$, that is $r$ is such that $\tilde{\Omega}=r^{N}\left(\Omega \cap B_{S}\right)$ with $S=2-2^{-\bar{k}} \leq 2$. Let $x_{0}$ be such that $B_{1}\left(x_{0}\right)$ is a minimizing ball for $\mathcal{A}(\tilde{\Omega})$. Then, recalling that $b_{\bar{k}}=\left|B_{1}\right|^{-1}\left|\Omega \backslash B_{S}\right| \leq$ $C(N) D(\Omega)$,

$$
\begin{aligned}
\left|B_{1}\right| \mathcal{A}(\Omega) \leq & \left|\Omega \Delta B_{1}\left(\frac{x_{0}}{r}\right)\right| \leq\left|\Omega \backslash B_{S}\right|+\left|\left(\Omega \cap B_{S}\right) \Delta B_{1}\left(\frac{x_{0}}{r}\right)\right| \\
\leq & C D(\Omega)+\left|\left(\Omega \cap B_{S}\right) \Delta B_{\frac{1}{r}}\left(\frac{x_{0}}{r}\right)\right| \\
& +\left|B_{\frac{1}{r}}\left(\frac{x_{0}}{r}\right) \Delta B_{1}\left(\frac{x_{0}}{r}\right)\right| \\
\leq & C D(\Omega)+\frac{\left|B_{1}\right|}{r^{N}} \mathcal{A}(\tilde{\Omega})+\left|B_{1}\right|\left(1-\frac{1}{r^{N}}\right) \\
\leq & C D(\Omega)+\left|B_{1}\right| \mathcal{A}(\tilde{\Omega})+C(N) b_{\bar{k}} \\
\leq & C D(\Omega)+\left|B_{1}\right| \mathcal{A}(\tilde{\Omega}) .
\end{aligned}
$$

\section{Proof of Theorem 1.4}

In order to reduce it to Theorem 3.1, we need to start with a set which is already close to a ball. Thanks to Theorem 1.2, this can be achieved by assuming the deficit sufficiently small (the quantitative inequality being trivial in the other regime).

We have now all the ingredients to prove Theorem 1.4.

Proof of Theorem 1.4. First note that if $D(\Omega) \geq \delta_{0}$ then, since $\mathcal{A}(\Omega) \geq 2$,

$$
D(\Omega) \geq 4 \frac{\delta_{0}}{4} \geq \frac{\delta_{0}}{4} \mathcal{A}(\Omega)^{2} .
$$

Hence we can assume that $D(\Omega)$ is as small as we wish as long as the smallness depends only on $N$. We now take $\delta_{0}$ smaller than the constant $\delta$ in Lemma 6.2 and, assuming that $D(\Omega) \leq \delta_{0}$, we use Lemma 6.2 to find a set $\tilde{\Omega}$ with $\operatorname{diam}(\tilde{\Omega}) \leq d=$ $d(N)$ and satisfying all the properties there. In particular, up to a translation we can assume that $\tilde{\Omega} \subset B_{d}$. Up to choosing $\delta_{0}$ smaller we can apply Theorem 1.2 and Lemma 2.3 (ii) to ensure that $\alpha(\tilde{\Omega}) \leq \varepsilon_{0}$ where $\varepsilon_{0}=\varepsilon_{0}(N, d)=\varepsilon_{0}(N)$ is the 
constant appearing in the statement of Theorem 3.1. This, together with Lemma 2.3 , (i), grants that

$$
D(\tilde{\Omega}) \geq c(N) \alpha(\tilde{\Omega}) \geq c(N) \mathcal{A}(\tilde{\Omega})^{2} .
$$

Hence, by Lemma 6.2 and assuming that $\mathcal{A}(\Omega) \geq C D(\Omega)$ (since otherwise there is nothing to prove),

$$
D(\Omega) \geq c D(\tilde{\Omega}) \geq c A(\tilde{\Omega})^{2} \geq c \mathcal{A}(\tilde{\Omega})^{2} \geq c \mathcal{A}(\Omega)^{2}-C D(\Omega)^{2}
$$

from which the conclusion easily follows since $D(\Omega) \leq \delta_{0} \ll 1$.

\section{APPENDIX A.}

Here we put some inequalities that are used throughout the paper.

Lemma A.1 ([FZ16, Lemma 2.3]). Let $p>1$. There exists $c(p) \geq 0$ such that if $\kappa \geq 0$ and $\xi, \eta \in \mathbb{R}^{n}$ then

$$
\left(\left(\kappa^{2}+|\xi|^{2}\right)^{\frac{p-2}{2}} \xi-\left(\kappa^{2}+|\eta|^{2}\right)^{\frac{p-2}{2}} \eta\right) \cdot(\xi-\eta) \geq c\left(\kappa^{2}+|\xi|^{2}+|\eta|^{2}\right)^{\frac{p-2}{2}}|\xi-\eta|^{2} .
$$

Moreover, there exists another constant $C(p) \geq 0$ such that if $\Omega \subset \mathbb{R}^{n}$ is an open set and for $u, v \in W^{1, p}(\Omega)$ and $0 \leq s \leq 1$, we set $u^{s}(x)=s u(x)+(1-s) v(x)$, then the following two inequalities hold:

- for $p \geq 2$

$$
\int_{\Omega}|\nabla u-\nabla v|^{p} \leq C \int_{0}^{1} \frac{1}{s} d s \int_{\Omega}\left(\left|\nabla u^{s}\right|^{p-2} \nabla u^{s}-|\nabla v|^{p-2} \nabla v\right) \cdot \nabla\left(u^{s}-v\right) ;
$$

- for $1<p<2$

(A.2)

$$
\begin{aligned}
& \int_{\Omega}|\nabla u-\nabla v|^{p} \\
& \leq C\left(\int_{0}^{1} \frac{1}{s} d s \int_{\Omega}\left(\left|\nabla u^{s}\right|^{p-2} \nabla u^{s}-|\nabla v|^{p-2} \nabla v\right) \cdot \nabla\left(u^{s}-v\right)\right)^{\frac{p}{2}}\left(\int_{\Omega}(|\nabla u|+|\nabla v|)^{p}\right)^{1-\frac{p}{2}} .
\end{aligned}
$$

Lemma A.2. Let $x, y \in \mathbb{R}^{N}, p \in(1, \infty)$. Then the following inequalities hold:

- if $p \geq 2$, then

$$
|y|^{p} \geq|x|^{p}+p|x|^{p-2} x \cdot(y-x)+c|y-x|^{p}
$$

for some $c=c(p)>0$;

- if $1<p<2$, then

$$
|y|^{p} \geq|x|^{p}+p|x|^{p-2} x \cdot(y-x)+c|y-x|^{2}\left(|x|^{2}+|y-x|^{2}\right)^{\frac{p-2}{2}}
$$

for some $c=c(p)>0$.

Proof. Consider a function $f: \mathbb{R}^{N} \rightarrow \mathbb{R}$ defined as $f(x)=|x|^{p}$. Writing Taylor expansion for $f$ we get

$$
|y|^{p}=|x|^{p}+p|x|^{p-2} x \cdot(y-x)+\int_{0}^{1}(1-t) D^{2} f(x+t(y-x))(y-x) \cdot(y-x) d t .
$$


If $p=2$, we thus have

$$
|y|^{2}=|x|^{2}+2 x \cdot(y-x)+\frac{1}{2}|y-x|^{2},
$$

which gives us a desired inequality. We shall consider $p \neq 2$ from now on.

For $p \neq 2$ the Hessian $D^{2} f(x)$ looks as follows:

$$
D^{2} f(x)=p|x|^{p-2} I d+p(p-2)|x|^{p-4} A,
$$

where $A_{i, j}=x_{i} x_{j}$. We notice that

$$
0 \leq A \xi \cdot \xi \leq|x|^{2}|\xi|^{2} \text { for any vector } \xi \in \mathbb{R}^{N},
$$

yielding

$$
D^{2} f(x) \xi \cdot \xi \geq c|x|^{p-2}|\xi|^{2} \text { for any vector } \xi \in \mathbb{R}^{N},
$$

where $c=c(p)>0(c=p$ for $p>2, c=p(p-1)$ for $1<p<2)$.

So, we have

$$
|y|^{p} \geq|x|^{p}+p|x|^{p-2} x \cdot(y-x)+|y-x|^{2} \int_{0}^{1}(1-t)|x+t(y-x)|^{p-2} d t .
$$

Let us consider the cases of different $p$ separately. First, we deal with $1<p<2$. In this case $p-2<0$ and so

$$
\int_{0}^{1}(1-t)|x+t(y-x)|^{p-2} d t \geq \frac{1}{4} \int_{1 / 4}^{3 / 4}(|x|+t|y-x|)^{p-2} d t \geq c\left(|x|^{2}+|y-x|^{2}\right)^{\frac{p-2}{2}}
$$

finishing the proof of lemma in this case.

To tackle the case $p>2$, we further consider two cases. If $|y-x|<2|x|$, then

$$
\int_{0}^{1}(1-t)|x+t(y-x)|^{p-2} d t \geq c \int_{0}^{1 / 4}|x|^{p-2} d t \geq c|y-x|^{p-2} .
$$

If instead $|y-x| \geq 2|x|$, then

$$
\int_{0}^{1}(1-t)|x+t(y-x)|^{p-2} d t \geq c \int_{4 / 7}^{6 / 7}|y-x|^{p-2} d t \geq c|y-x|^{p-2} .
$$

\section{REFERENCES}

[AFM14] E. Acerbi, N. Fusco, and M. Morini. Minimality via second variation for a nonlocal isoperimetric problem. Comm.Math. Phys., 322:515-557, 2014.

[AAC86] N. Aguilera, H. W. Alt, and L. A. Caffarelli. An optimization problem with volume constraint. SIAM J. Control Optim., 24(2):191-198, 1986.

[ACF84] H. W. Alt, L. A. Caffarelli, and A. Friedman. A free boundary problem for quasi-linear elliptic equations. Annali della Scuola Normale Superiore di Pisa-Classe di Scienze, 4(11):1-44, 1984.

[AC81] H. W. Alt and L. A. Caffarelli. Existence and regularity for a minimum problem with free boundary. J. Reine Angew. Math., 325:105-144, 1981.

[ATW93] F. Almgren, J. E. Taylor, and L. Wang. Curvature-driven flows: a variational approach. SIAM J. Control Optim., 31(2):387-438, 1993.

[BDPV15] L. Brasco, G. De Philippis, and B. Velichkov. Faber-krahn inequalities in sharp quantitative form. Duke Math. J., 164(9):1777-1831, 2015. 
[BZ88] J. E. Brothers and W. P. Ziemer. Minimal rearrangements of Sobolev functions. J. reine angew. Math, 384:153-179, 1988.

[CL12] M. Cicalese and G. P. Leonardi. A selection principle for the sharp quantitative isoperimetric inequality. Arch. Ration. Mech. Anal., 206:617-643, 2012.

[DP05] D. Danielli, A. Petrosyan. A minimum problem with free boundary for a degenerate quasilinear operator. Calculus of Variations and Partial Differential Equations, 23:97124, 2005.

[DPMM19] G. De Philippis, M. Marini, and E. Mukoseeva. The sharp quantitative isocapacitary inequality. arXiv:1901.11309, accepted to RMI, 2019.

[FMP08] N. Fusco, F. Maggi, and A. Pratelli. The sharp quantitative isoperimetric inequality. Ann. Math., 168:941-980, 2008.

[FMP09] N. Fusco, F. Maggi, and A. Pratelli. Stability estimates for certain faber-krahn, isocapacitary and cheeger inequalities. Annali della Scuola Normale Superiore di Pisa-Classe di Scienze, 8:51-71, 2009.

[Fug89] B. Fuglede. Stability in the isoperimetric problem for convex or nearly spherical domains in $\mathbb{R}^{n}$. Trans. Amer. Math. Soc., 314:619-638, 1989.

[FZ16] N. Fusco, Y. R. Y. Zhang. A quantitative form of the Faber-Krahn inequality. preprint, https://cvgmt.sns.it/paper/3015/, 2016.

[FZ17] N. Fusco, Y. R. Y. Zhang. A quantitative form of the Faber-Krahn inequality. Calculus of Variations and Partial Differential Equations, 56(5), 2017.

[GM] M. Giaquinta and L. Martinazzi. An Introduction to the Regularity Theory for Elliptic Systems, Harmonic Maps and Minimal Graphs, volume 11 of Lecture Notes (Scuola Normale Superiore). Edizioni della Normale, 2012.

[G] E. Giusti. Direct Methods in the Calculus of Variations. WSPC, 2003.

[Gr] P. Grisvard. Elliptic problems in nonsmooth domains, volume 24 of Monographs and Studies in Mathematics. Pitman (Advanced Publishing Program), Boston, MA, 1985.

[HHW91] R. R. Hall, W. K. Hayman, and A. W. Weitsman. On asymmetry and capacity. J. d'Analyse Math., 56:87-123, 1991.

[HN92] W. Hansen and N. Nadirashvili. Isoperimetric inequalities for capacities. In Harmonic analysis and discrete potential theory (Frascati, 1991), pages 193-206. Plenum, New York, 1992.

[HP05] A. Henrot and M. Pierre. Variation et optimisation de formes:Une analyse géométrique, volume 48 of Mathématiques et Applications. Springer-Verlag Berlin Heidelberg, 2005.

[L06] P. Lindqvist. Notes on the p-Laplace equation. newblock Univ. Jyväskylä, Report 102, 2006.

[L88] G. M. Lieberman. Boundary regularity for solutions of degenerate elliptic equations. Nonlinear Anal., 12:1203-1219, 1988.

[SZ] J. Sokolowski and J. P. Zolésio Introduction to Shape Optimization: Shape Sensitivity Analysis, volume 16 of Springer Series in Computational Mathematics. Springer-Verlag Berlin Heidelberg, 1992.

[T67] N. Trudinger. On Harnack Type Inequalities and Their Application to Quasilinear Elliptic Equations. Communications on Pure and Applied Mathematics, 20:721-747, 1967.

E.M: Department of Mathematics and Statistics, P.O. Box 68 (Gustaf Hällströmin Katu 2), FI-00014 University of Helsinki, Finland

Email address: ekaterina.mukoseeva@helsinki.fi 